\title{
APPLIED MATHEMATICS
}

AT THE U.S. DEPARTMENT OF ENERGY: Past, Present and a View to the Future

A Report by an Independent Panel from the Applied Mathematics Research Community

May 2008

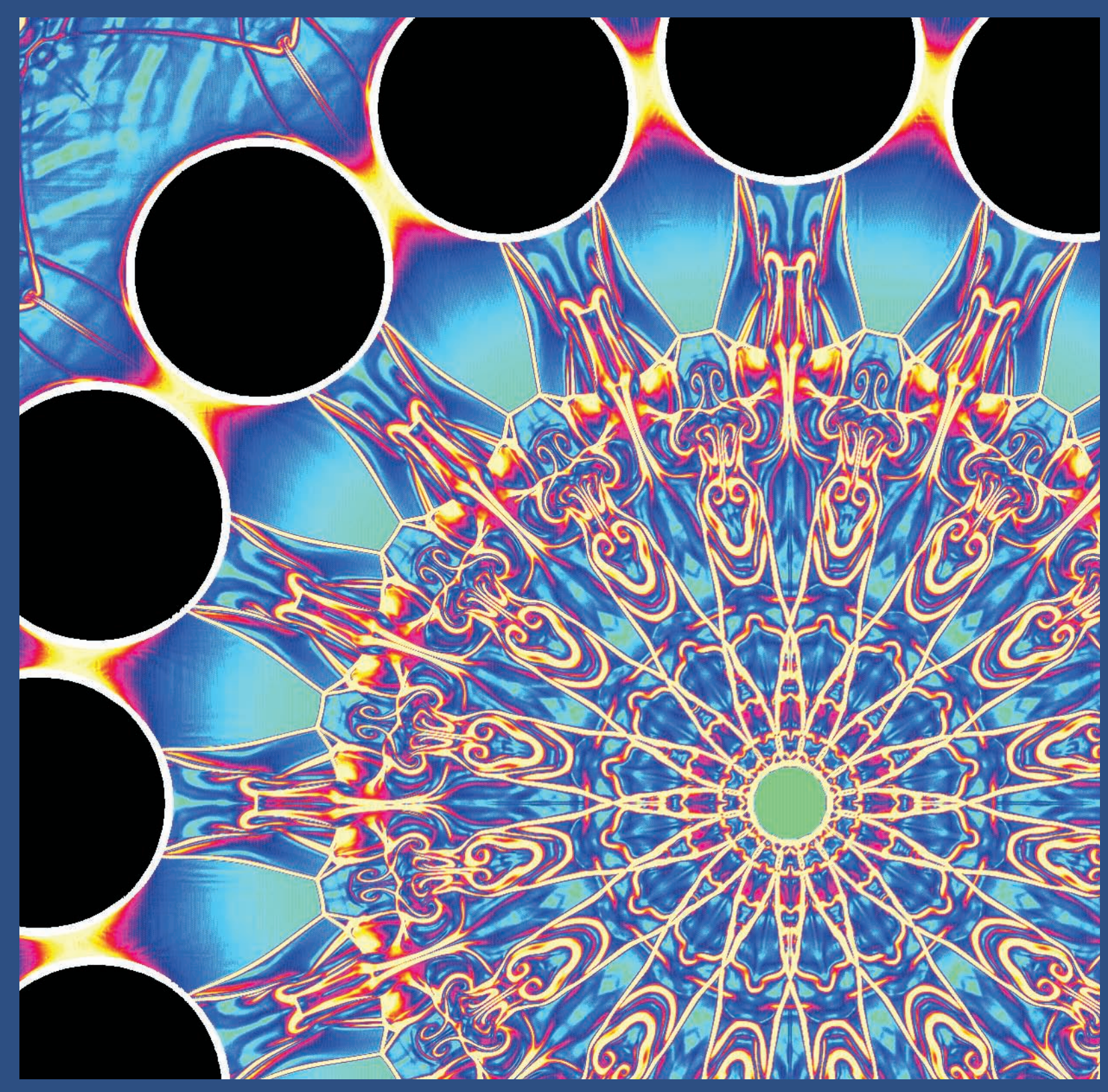




\section{About the Cover}

A cylindrical shock wave produces this intricate flow pattern as it converges from outside sixteen circular obstacles and diffracts. Researchers are interested in the structure of the solution near the focus (green region). This high-resolution numerical solution was computed using adaptive overlapping meshes. Source: Center for Applied Scientific Computing, Lawrence Livermore National Laboratory.

\section{Disclaimer}

This document was prepared as an account of work sponsored by an agency of the United States government. Neither the United States government nor Lawrence Livermore National Security, LLC, nor any of their employees makes any warranty, expressed or implied, or assumes any legal liability or responsibility for the accuracy, completeness, or usefulness of any information, apparatus, product, or process disclosed, or represents that its use would not infringe privately owned rights. Reference herein to any specific commercial product, process, or service by trade name, trademark, manufacturer, or otherwise does not necessarily constitute or imply its endorsement, recommendation, or favoring by the United States government or Lawrence Livermore National Security, LLC. The views and opinions of authors expressed herein do not necessarily state or reflect those of the United States government or Lawrence Livermore National Security, LLC, and shall not be used for advertising or product endorsement purposes.

LLNL-TR-401536 


\section{APPLIED MATHEMATICS AT THE U.S. DEPARTMENT OF ENERGY: Past, Present and a View to the Future}

A Report by an Independent Panel from the Applied Mathematics Research Community

May 2008

\section{Panel Members:}

David L. Brown (chair), Lawrence Livermore National Laboratory John Bell, Lawrence Berkeley National Laboratory Donald Estep, Colorado State University

William Gropp, University of Illinois Urbana-Champaign

Bruce Hendrickson, Sandia National Laboratories

Sallie Keller-McNulty, Rice University

David Keyes, Columbia University

J. Tinsley Oden, The University of Texas at Austin

Linda Petzold, University of California, Santa Barbara

Margaret Wright, New York University 



\section{Table of Contents}

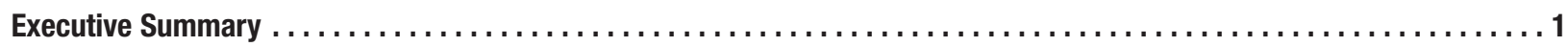

1. Applied Mathematics for the Department of Energy $\ldots \ldots \ldots \ldots \ldots \ldots \ldots \ldots \ldots \ldots \ldots \ldots \ldots \ldots \ldots \ldots \ldots \ldots$

1.1 DOE Mission Drivers and Mathematical Challenges $\ldots \ldots \ldots \ldots \ldots$

1.2 Historical Successes of the DOE Applied Mathematics Program . . . . . . . . . . . . . .

1.3 Future Success of the DOE Applied Mathematics Program . . . . . . . . . . . . . . .

2. Advancing Mathematics for Modeling, Simulation, Analysis and Understanding of Complex Systems . . . . . . . . . 9

2.1 Predictive Modeling and Simulation of Complex Systems . . . . . . . . . . . . . 10

2.1.1 Multiscale, Multiphysics, and Complex Hybrid Models . . . . . . . . . . . . . . . 11

2.1.2 The Role of Data-Model Fusion in Prediction . . . . . . . . . . . . . . . . . . 12

2.1.3 Modeling Stochastic Effects in Complex Systems . . . . . . . . . . . . . . . . . . 14

2.1.4 Networks, Systems and Systems of Systems . . . . . . . . . . . . . . . . 15

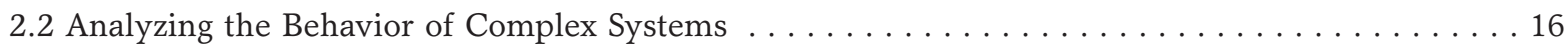

2.2.1 The Role of Data in the Analysis and Understanding of Complex Systems . . . . . . . . 16

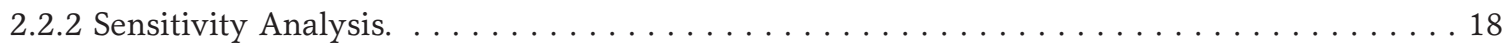

2.2 .3 Uncertainty Quantification and Mitigation. . . . . . . . . . . . . . . . 19

2.3 Using Complex Systems to Inform Policy-Making . . . . . . . . . . . . . . . . . 20

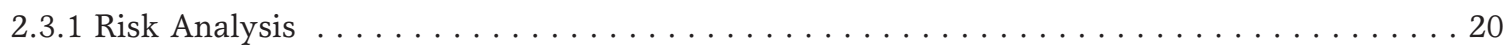

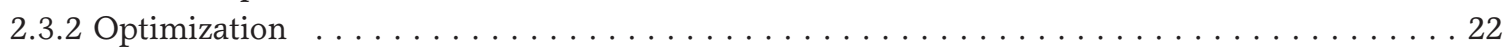

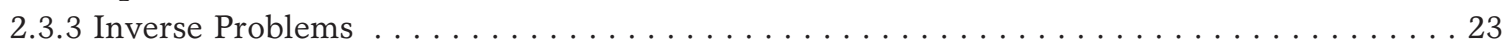

3. Driving Innovation and Discovery in Applied Mathematics Through Effective Program Leadership . . . . . . . . . . 25

3.1. Encouraging and Rewarding Risk-Taking in Research . . . . . . . . . . . . . . . . . 25

3.2. Building Effective Connections with Science and Engineering. . . . . . . . . . . . . 25

3.3. Leveraging the Expertise in National Laboratories and Universities . . . . . . . . . . . . . 26

3.4. Connecting Applied Mathematics and Advanced Computing. . . . . . . . . . . . 26

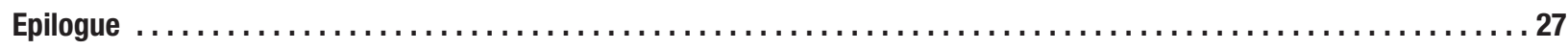

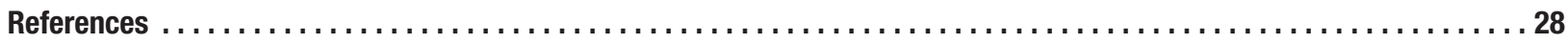





\section{Executive Summary}

Over the past half-century, the Applied Mathematics program in the U.S. Department of Energy's Office of Advanced Scientific Computing Research has made significant, enduring advances in applied mathematics that have been essential enablers of modern computational science.

Motivated by the scientific needs of the Department of Energy and its predecessors, advances have been made in mathematical modeling, numerical analysis of differential equations, optimization theory, mesh generation for complex geometries, adaptive algorithms and other important mathematical areas. High-performance mathematical software libraries developed through this program have contributed as much or more to the performance of modern scientific computer codes as the high-performance computers on which these codes run. Funding from this program has assisted generations of graduate and postdoctoral students who have continued on to populate and contribute productively to research organizations in industry, universities and federal laboratories. The combination of these mathematical advances and the resulting software has enabled high-performance computers to be used for scientific discovery in ways that could only be imagined at the program's inception.

Our nation, and indeed our world, face great challenges that must be addressed in coming years, and many of these will be addressed through the development of scientific understanding and engineering advances yet to be discovered. The U.S. Department of Energy (DOE) will play an essential role in providing science-based solutions to many of these problems, particularly those that involve the energy, environmental and national security needs of the country. As the capability of highperformance computers continues to increase, the types of questions that can be answered by applying this huge computational power become more varied and more complex. It will be essential that we find new ways to develop and apply the mathematics necessary to enable the new scientific and engineering discoveries that are needed.

In August 2007, a panel of experts in applied, computational and statistical mathematics met for a day and a half in Berkeley, California to understand the mathematical developments required to meet the future science and engineering needs of the DOE. It is important to emphasize that the panelists were not asked to speculate only on advances that might be made in their own research specialties. Instead, the guidance this panel was given was to consider the broad science and engineering challenges that the DOE faces and identify the corresponding advances that must occur across the field of mathematics for these challenges to be successfully addressed. As preparation for the meeting, each panelist was asked to review strategic planning and other informational documents available for one or more of the DOE Program Offices, including the Offices of Science, Nuclear Energy, Fossil Energy, Environmental Management, Legacy Management, Energy Efficiency \& Renewable Energy, Electricity Delivery \& Energy Reliability and Civilian Radioactive Waste Management as well as the National Nuclear Security Administration. The panelists reported on science and engineering needs for each of these offices, and then discussed and identified mathematical advances that will be required if these challenges are to be met.

A review of DOE challenges in energy, the environment and national security brings to light a broad and varied array of questions that the DOE must answer in the coming years. A representative subset of such questions includes:

- Can we predict the operating characteristics of a clean coal power plant?

- How stable is the plasma containment in a tokamak?

- How quickly is climate change occurring and what are the uncertainties in the predicted time scales?

- How quickly can an introduced bio-weapon contaminate the agricultural environment in the US?

- How do we modify models of the atmosphere and clouds to incorporate newly collected data of possibly of new types?

- How quickly can the United States recover if part of the power grid became inoperable? 
- What are optimal locations and communication protocols for sensing devices in a remote-sensing network?

- How can new materials be designed with a specified desirable set of properties?

In comparing and contrasting these and other questions of importance to DOE, the panel found that while the scientific breadth of the requirements is enormous, a central theme emerges: Scientists are being asked to identify or provide technology, or to give expert analysis to inform policy-makers that requires the scientific understanding of increasingly complex physical and engineered systems. In addition, as the complexity of the systems of interest increases, neither experimental observation nor mathematical and computational modeling alone can access all components of the system over the entire range of scales or conditions needed to provide the required scientific understanding.

These observations motivated the panel to articulate a new framework for describing the applied mathematical developments that will be required to address future scientific and engineering challenges for DOE. Two significant reasons make this new framework valuable. The first is that it aligns the thinking about future mathematics research for DOE around the most difficult scientific and engineering problems that the DOE faces in coming years. The second is that it identifies needed mathematical advances in a way that clearly articulates how current strengths in the DOE Applied Mathematics program can be leveraged and, perhaps more importantly, where significant gaps exist in the mathematics that must be developed to understand these complex systems. Expressed in terms of applied mathematics research topics, these findings can be summarized as follows:

- DOE must leverage the current strengths of the Applied Mathematics program in predictive simulation and modeling, expanding these capabilities to more fully address simulation and modeling for complex systems. Areas that are not adequately developed include the development and analysis of methods to model large stochastic systems, and techniques for decomposing complex systems into systems of canonical subsystems. The mathematical underpinnings of sensitivity analysis, uncertainty quantification, risk analysis, optimization and inversion must also be significantly expanded to address the challenges presented by complex systems.

- It is essential that the program increasingly focus its perspective on the end goal of developing mathematical approaches for understanding the complex systems themselves. This recognizes that breaking problems down into simpler components cannot be the only mathematical approach, but must be complemented with the development of modeling, simulation and analysis tools that deal with the full complex systems.

- The mathematics for analyzing very large amounts of data, whether produced by simulations or through experimental observations, requires serious development. A particular challenge is to enhance the theory and tools for data-model fusion for complex systems, where observational or experimental data are incorporated in an essential way with simulation and modeling.

The full panel report describes these challenges in terms of three encompassing themes together with high-level strategies for addressing the gaps in our understanding. These are summarized below:

\section{Predictive modeling and simulation of complex systems}

Advance the fidelity, predictability and sophistication of modeling and simulation methodologies for complex systems:

- Develop analytical and computational approaches needed to understand and model the behavior of complex multiphysics, and multiscale phenomena.

- Enhance the theory and tools for complex multiscale, multicomponent models when observational or experimental data are incorporated in an essential way.

- Develop new approaches for efficient modeling of large stochastic systems.

- Develop mathematical techniques for decomposing complex systems into systems of canonical subsystems and modeling their behavior. 


\section{Mathematical analysis of the behavior of complex systems}

Address the challenges of analyzing and understanding the behavior of mathematical models for complex scientific and engineering systems:

- Develop sound, computationally feasible strategies and methods for the collection, organization, statistical analysis and use of data associated with complex systems.

- Advance the theory and tools for sensitivity analysis to address the challenges posed by complex multiscale, multicomponent models.

- Significantly advance the theory and tools for quantifying the effects of uncertainty and numerical simulation error on predictions using complex models and when fitting complex models to observations.

\section{Using models of complex systems to inform policy makers}

Develop the mathematics needed to inform policy makers based on the prediction, optimization and understanding of complex systems:

- Significantly advance the mathematics that supports risk analysis techniques for policy-making involving complex systems that include natural and engineered components, and economic, security and policy consequences.

- Develop techniques for formulating, analyzing and solving challenging optimization problems arising in complex natural and engineered systems.

- Develop techniques for addressing the mathematical and computational difficulties of inverse problems associated with complex systems.
In addition to developments in fundamental mathematics in these areas, the program must continue to develop the high-performance software that translates mathematical advances for complex systems into enabling computational tools that can be used by computational scientists. This will also depend on complementary investments focused on understanding and exploiting the latest high-performance hardware developments as they are anticipated and become available.

Finally, the panel paid special attention to the opportunities that exist for innovative approaches in program leadership that can promote innovation and discovery in applied mathematics. The panel report recommends that the program encourage risk-taking and innovation in research by taking steps that reduce the perceived risk to investigators while at the same time ensuring accountability for their research activities. Rewarding investigators who consistently produce innovative and mission-relevant research results with continued long-term support encourages risk-taking and recognizes that "transformational" science is often the result of the concentrated long-term development of understanding. Introducing new ideas into research efforts is also an essential element of a successful program. This can be enhanced by providing opportunities for young researchers to participate in the program, and for researchers to interact and collaborate in intense environments such as workshops and summer schools. Finally, the effective application of mathematics requires deep understanding of the underlying scientific and engineering applications. By encouraging and supporting early interaction and collaboration of mathematicians with their colleagues in science and engineering fields, the program will accelerate the development of successful applications of mathematics to the scientific and engineering challenges that the DOE must address for the future. 


\section{Applied Mathematics for the Department of Energy}

\subsection{DOE mission drivers and mathematical challenges}

As the U.S. Department of Energy (DOE) works to meet its energy, environmental and national security missions, increasingly complex scientific and technological challenges must be addressed. New energy technologies will be required both for tapping the potential of new sources of energy and for effectively utilizing existing energy resources. Policy makers will need to understand quantitatively the impact of energy policies on the environment and be able to evaluate the risks associated with different strategies for waste storage and environmental cleanup. DOE must also ensure the safety and reliability of the nuclear stockpile while preventing the proliferation of nuclear materials ${ }^{1}$.

Applied mathematics has an increasingly important role to play in the support of these expanding scientific and technological challenges. Where simple models, simple approximations and one-off solutions once sufficed, the need now exists to develop, simulate, analyze and understand models for complex combinations of processes with multiple scales in space and time $e^{2,3,4,5}$. Policy makers need science-based analyses of these complex models, supported by clearly defined margins of uncertainty and statistical characterizations. When observational or experimental measurements are available, these must be incorporated with mathematical rigor into the scientific analyses that support the creation of critical national policy. The purpose of this report is to provide a description of the applied mathematics advances that will be required to help answer the many mission-critical questions that DOE faces in the coming years.

Although the issues associated with meeting the DOE mission span an enormous range of applications, from a mathematical perspective a number of specific themes emerge. Next-generation nuclear power plants ${ }^{6}$, fusion reactors ${ }^{7,8}$, clean coal technologies and new engine designs all require an ability to model systems that incorporate a variety of physical processes across a broad range of scales. ${ }^{9}$ Carbon sequestration, enhanced oil recovery, nuclear waste storage and environmental cleanup problems all require high-fidelity models for subsurface flow that integrate the inherent uncertainties in subsurface characterization with an integrated capability for risk management ${ }^{10}$. Climate modeling involves a number of similar considerations and introduces additional elements of data assimilation to integrate observational data directly into the simulation process.

Other mission-critical applications require substantial improvements in the ability to mathematically model behavior at microscopic or quantum mechanical scales. The design of systems to harness biochemical processes for the production of biofuels requires new approaches for modeling excited states of chemical systems ${ }^{11}$. The design of new materials at the nanoscale, such as high temperature superconductors, requires new, more fundamental approaches to computing the properties of materials from first principles ${ }^{12}$. Understanding the behavior of biological systems at microscopic scales requires methodologies for capturing the effect of fluctuations in nonequilibrium thermodynamic systems.

A class of applications of emerging importance to DOE focuses on the behavior of complex networks. Typical of this type of problem are issues of reliability and security of the nation's electrical power grid. In this case, the system is a hierarchical network with independent entities controlling local subnets. System-level models at scales ranging from individual power plants to the international energy distribution systems raise problems in policy-making and risk analysis. Another example involves the logistical issues arising in the transportation of nuclear waste, which are characterized by discrete optimization problems ${ }^{13}$.

Data are no longer simple and researchers find themselves grappling with ever-increasing amounts of data ${ }^{14}$. Today, DOE applications such as climate modeling can bring massive amounts of heterogeneous data together that must be understood both alone and in the context of complex simulations. High-energy physics experiments will soon produce enormous amounts of data that must be managed, transmitted and analyzed. Biology is becoming an 


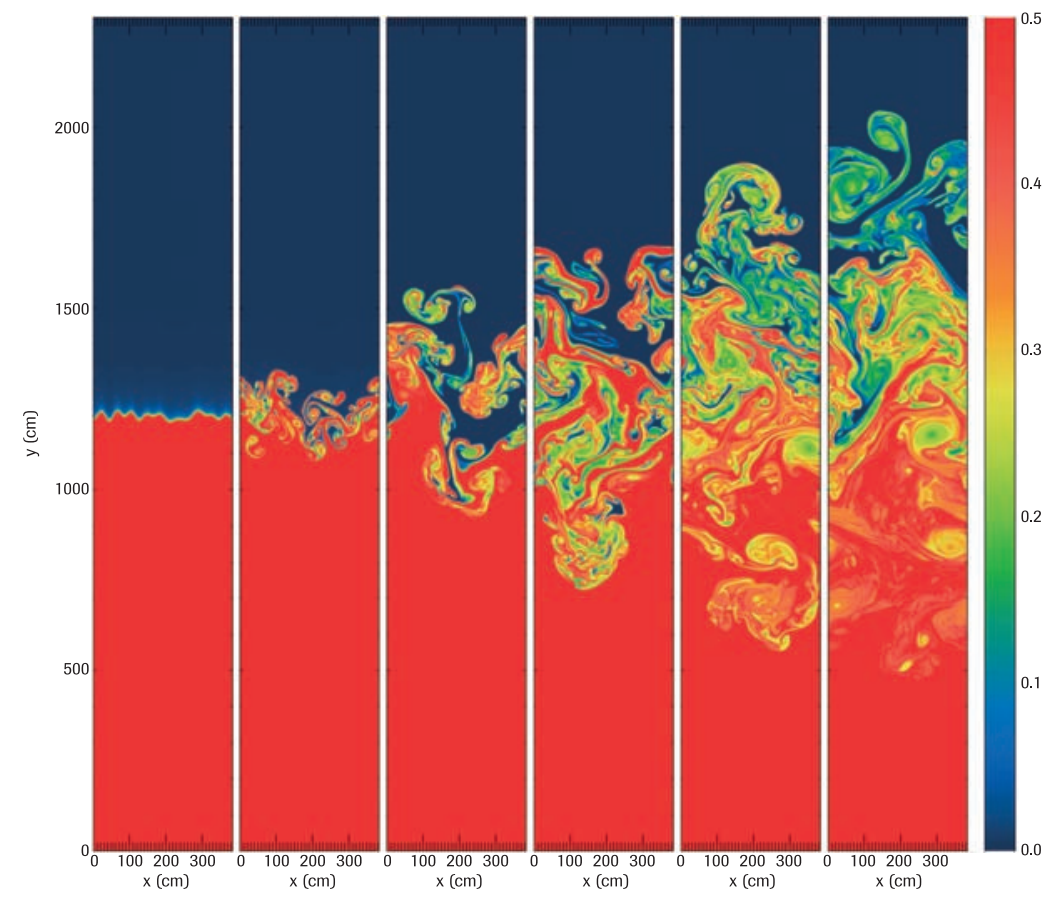

FIGURE 1. Accelerating a thermonuclear flame to a large fraction of the speed of sound (possibly supersonic) is one of the main difficulties in modeling Type la supernova explosions, which likely begin as a nuclear runaway near the center of a carbon-oxygen white dwarf. Algorithms developed under the auspices of the DOE Applied Mathematics program were used to perform this simulation showing an unstable outward propagating flame. Source: Center for Computational Science and Engineering, Lawrence Berkeley National Laboratory increasingly quantitative science with the advent of unprecedented high-throughput experimental measurement techniques, and correspondingly the ability to digest and analyze huge amounts of heterogeneous data has become essential for scientific discovery in the biological arena. It is therefore crucial that sound strategies and methods be developed for the collection, organization, analysis, and representation of the results for extremely heterogeneous and diverse sources of information. These methodologies must integrate the diverse sources of data and information and their associated uncertainties to develop full distributions for performance metrics that can aid DOE policy-making in the face of uncertainty.

Each of the problem areas discussed above is complex and multifaceted and will require collaborations that encompass a broad range of expertise. Each solution is a multi-step process embodying a number of elements. In many cases new and appropriate mathematical models need to be formulated. These models must incorporate the appropriate coupling between disparate length and time scales and between fundamentally different types of processes, and may also need to incorporate relevant experimental or observational data. Some type of solution or approximation procedure needs to be developed to quantify the behavior of the model.
This quantification should include a characterization of inherent uncertainties in the model. The behavior of the model must be analyzed in the context of available data that characterizes the observed behavior of the system. This type of analysis can range from statistical behavior of simulation data to the solution of inverse problems to dynamic data assimilation that integrates simulation with observation. Finally, the models that have been developed can be used for design optimization, risk analysis and policy-making.

Applied mathematics has a substantial role to play in all aspects of the solution process. The DOE Applied Mathematics Program has traditionally played a strong role in the development of numerical methods for differential equations, numerical linear algebra and optimization. Developing methodologies for solving the classes of problems discussed above will certainly build on that expertise; however, applied mathematics must be involved more broadly in the overall solution strategy. Applied mathematicians must work collaboratively with domain scientists and engineers in the entire process from problem formulation through the analysis and integration of data to the design/ policy-making process. New mathematical approaches will be required at each step in the solution process to adequately address DOE mission requirements. 


\subsection{Historical successes of the DOE Applied Mathematics program}

The use of mathematical and computational models to simulate physical events or the behavior of engineered systems is arguably one of the most important developments in science and technology of the past century. Today, computational models based on mathematical characterizations of theory enable scientists and engineers to predict the behavior of extremely complex natural and human-made systems and provide a basis for creating policy critical to the competitiveness and well being of the nation. The DOE Applied Mathematics program has made substantial contributions to this development.

The growth over the past decades in the raw processing speed of computer processing units has been staggering. However, as impressive as this growth in computing power has been, our modern scientific computing capability would not have developed without an equally important investment in the underlying enabling mathematics. John von Neumann recognized this essential factor, and in the early 1950s, having become a Commissioner for the U. S. Atomic Energy Commission, asked Los Alamos computer scientist John R. Pasta to create a contract research program for applied mathematics and computer science. This AEC program, which funded research mathematicians and computer scientists at the AEC Laboratories and at U.S. universities, was the beginning of the present-day DOE

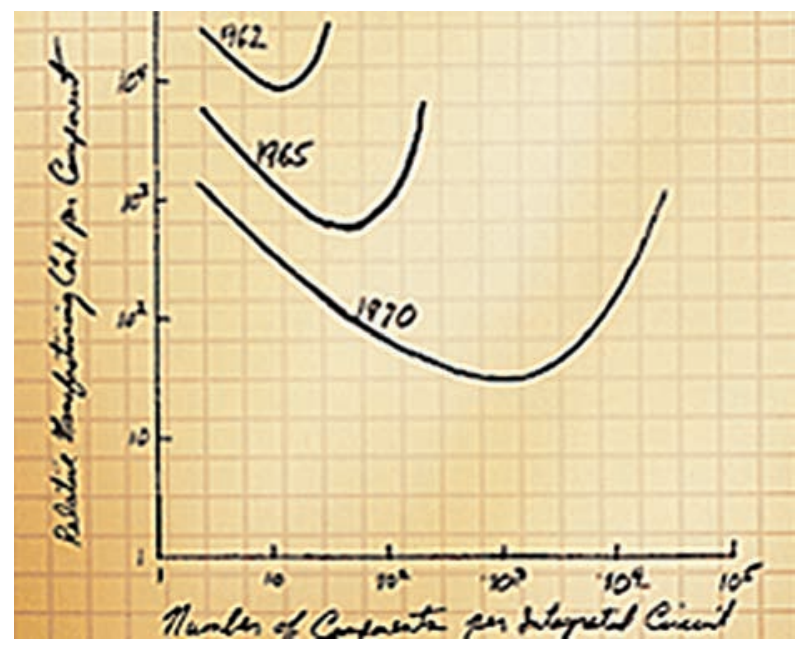

FIGURE 2. Moore's original 1965 graph showing the cost per transistor as a function of the number of transistors on a chip. Moore predicted that the number of transistors at the curve minimum, which represents the most efficient chip capacity, would double every two years.
Applied Mathematics program. During its 50 years of existence, the Applied Mathematics program has been responsible for supporting fundamental mathematical developments that have substantially advanced research in many scientific fields. The strength of these mathematical results derives not only from the development of algorithms that translate scientific theory into discrete equations that a computer can solve, but in the mathematical and numerical analysis that provides the basic understanding of both the scientific theories and their numerical counterparts.

The impact of the Applied Mathematics program on the field of fluid dynamics is one significant example of these contributions. In the early 1950s, researchers were interested in fluid behavior in regimes that allowed the formation of shocksvery rapid transitions in fluid velocity and pressure. Motivated by the need to understand and simulate these shock physics problems, Peter Lax and his colleagues at the Courant Institute of Mathematical Sciences began to look in more detail at the theory of hyperbolic systems of conservation laws, the category of nonlinear partial differential equations that describes the behavior of shocks and other wave-like phenomena in physics. A substantial body of work, much of it funded by the Applied Mathematics program, was summarized by Lax in his 1973 SIAM monograph entitled "Hyperbolic Systems of Conservation Laws and the Mathematical Theory of Shock Waves. ${ }^{15 " ~ L a x ~ p r o v i d e d ~ u s ~ w i t h ~ a n ~}$ elegant description of the theory for generalized solutions to the initial value problem for nonlinear hyperbolic systems that answers many of the basic structural questions about solutions to shock problems. This theory provided the foundation needed for applied mathematicians in the 1970s and 1980s to develop sophisticated new methods for solving systems of conservation laws that form the core of most modern compressible flow simulation codes used at universities and at DOE and other government research institutions. Additional mathematical developments, also funded in part by the Applied Mathematics program, established the framework to extend these types of approaches to a broader range of problems, including subsurface flow, combustion and atmospheric modeling.

The DOE Applied Mathematics program has also had significant impact on the field of mathematical optimization. For more than 30 years, DOE 


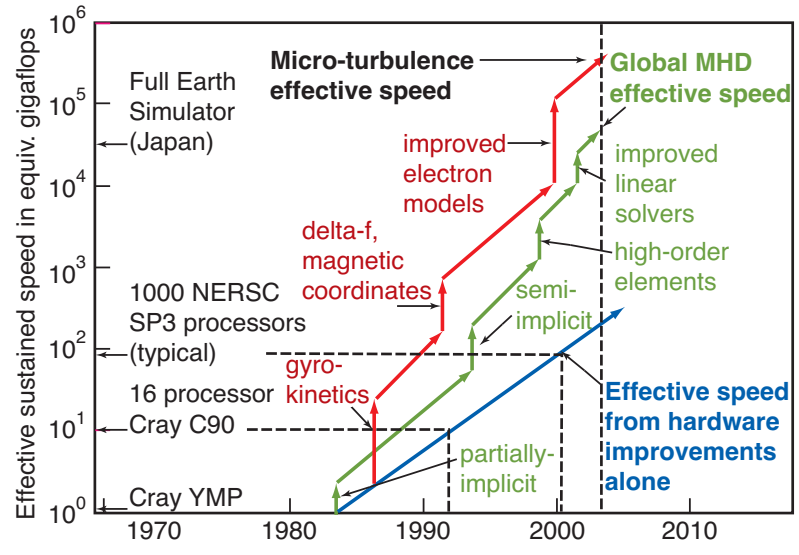

FIGURE 3. Magnetic fusion energy "effective speed" increases came from faster hardware, better mathematical models and improved computational algorithms. Source: A Science-Based Case for Large-Scale Simulation (SCaLeS) Volume 2

has supported research on a broad spectrum of topics in optimization, including theory and software development, with emphasis on their fruitful interplay. Researchers at Argonne National Laboratory, in collaboration with university colleagues, developed a substantial body of theory that was immediately, and still is, regarded as the foundation of nonlinear optimization. This theory in turn led to the development of the unconstrained optimization methods that form the mainstay of modern optimization. An integral part of the Argonne group's accomplishments was software based on sound mathematical theory. The MINPACK-1 package was among the first to include reliable routines for unconstrained optimization, nonlinear equations and nonlinear least squares. Another group at Stanford University developed both theory and software for constrained optimization problems with an emphasis on explaining the behavior in practice of the implemented methods. All of this software has been used to solve problems from a variety of disparate applications, including the optimal power flow problem, real-time optimization of a hydroelectric plant, optimal control in networks, calculation of beam emittances in a particle accelerator, and trajectory optimization.

\subsection{Future success of the DOE Applied Mathematics program}

Fluid dynamics and optimization are just two examples of areas on which this program has had enormous impact. Similar stories can be told about the advances in numerical linear algebra, dynamical systems, bifurcation theory, and many other areas. Future success of the program will be tied to our ability to make substantial investments to reach the vision of using high-performance-computing-based predictive simulation and analysis of complex natural and engineered systems as a basis for policy-making involving critical energy, environmental and national security issues. These compelling opportunities for new mathematical and statistical approaches to DOE's research challenges are predicated upon continued strength in fundamental areas of applied and computational mathematics. Areas such as the solution of ordinary differential equations, computational geometry, adaptive meshing, harmonic analysis, graph algorithms, and linear algebraic systems have made major progress in past decades in both theory and high-quality software. Nonetheless, there is a clear need for continued development of these and other more mature mathematical areas at their frontiers to meet the vision just articulated.

The next chapter examines DOE's future requirements in detail and identifies some of the prototypical problem areas where new research in applied mathematics can significantly advance our ability to find scientific and engineering solutions to pressing problems of national interest. From this survey of problems we extract a number of fundamental mathematical areas where new mathematical research is needed and identify specific research opportunities that would significantly advance our capabilities in these areas. These advancements can, in turn, help meet mission-critical needs throughout DOE. In the final chapter we identify some of the guiding principles for ensuring the effectiveness of a research program in applied mathematics and maximizing its impact on DOE mission problems. 


\section{Advancing Mathematics for Modeling, Simulation, Analysis and Understanding of Complex Systems}

For centuries, mathematics has provided the language for expressing scientific theories that describe how nature behaves. Now, mathematicallybased computational models enable scientists to move beyond using mathematics for its descriptive capabilities alone. Modern applied mathematics enables the prediction and understanding of the behavior of extremely complex natural and humanmade systems and provides a basis for creating policy critical to the competitiveness and well being of the nation.

For the discussion below, it will be useful to have a common understanding of what we mean by a "complex system." A complex system is a collection of multiple processes, entities or nested subsystems where the overall system is difficult to understand and analyze because of the following properties:

- The system components do not necessarily have mathematically similar structures and may involve different scales in time or space;

- The number of components may be large, sometimes enormous;

- Components can be connected in a variety of different ways, most often nonlinearly and/or via a network. Furthermore, local and systemwide phenomena may depend on each other in complicated ways;

- The behavior of the overall system can be difficult to predict from the behavior of individual components. Moreover, the overall system behavior may evolve along qualitatively different pathways that may display great sensitivity to small perturbations at any stage.

Such systems are often described as "multicomponent systems," or when the components are physics based, "multiphysics systems." When the components involve multiple spatial or temporal scales, the adjective "multiscale" can be used as well.

We also mean to discuss complex systems in the broadest possible sense. Examples of systems that we view to be complex include:

- Problems that involve a single physical system, which becomes complex when modeled using a multiscale approach. An example occurs in the composite design of materials when a hybrid discrete-continuum model is used to describe atomistic-macroscopic phenomena.

- Problems that involve the coupling of multiple physical processes described with different models. An example occurs in the modeling of carbon sequestration, where a quantitative study may require the simulation of multiple fluid phases, geomechanics, and a complex set of biogeochemical reactions over a wide range of scales.

- Problems that describe complex engineered systems. An example is the electric power grid, where models may involve inequality and other types of constraints, severe nonlinearities and discontinuities, a mixture of continuous and integer variables, a large number of variables, a huge range of scales, and non-unique solutions that may make it difficult to characterize the most physically reasonable result.

Careful observation has always been the mainstay of scientific and engineering discovery. With
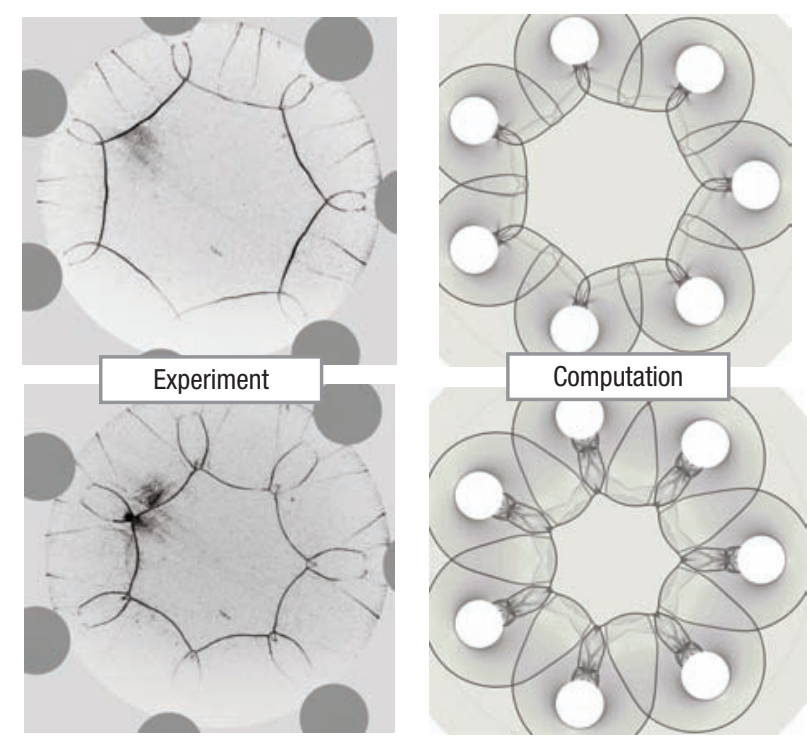

FIGURE 4. Understanding complex systems will require a combination of experimental and computational techniques. Left: experimental images of the diffraction pattern from a cylindrically converging shock that hits seven obstacles. Right: images from an adaptive numerical simulation, showing excellent agreement with the experiment. Source: Center for Applied Scientific Computing, Lawrence Livermore National Laboratory 
the development of modern mathematical and computational models for complex systems, computational prediction now complements detailed experiment and observation as a tool for developing scientific understanding. However, as the systems we are interested in become ever more complex, neither experimental observation nor mathematical and computational modeling alone can access all components of a complex system over the entire range of relevant scales. Reductionist approaches that seek to obtain understanding by breaking complex systems into simpler components may miss emergent, and often significant behavior that arises due to the interaction of these components in nonlinear ways. Researchers are now recognizing that through the fusion of observational data and predictive model-based simulation, a much fuller picture of the behavior of complex systems can be obtained. The "glue" that can provide this "datamodel fusion" is based on applied, statistical and computational mathematics.

The scientific and technical issues that DOE must address over the coming decades pose significant challenges. The remainder of this chapter discusses the advanced predictive tools needed to support effective policy-making by our nation's leaders. These tools will require new, rigorously justified mathematical developments in predictive modeling, simulation, analysis and understanding of complex systems.

\subsection{Predictive modeling and simulation of complex systems}

Advance the fidelity, predictability and sophistication of modeling and simulation methodologies by developing the mathematical tools needed for the analysis and simulation of complex systems characterized by combinations of multiple length and time scales, multiple processes or components.

The basis for policy-making based on predicting and understanding complex systems depends in an essential way on the development of mathematically rigorous, scientifically based models for those systems. This section discusses the mathematical developments that will be required to build multiscale, multiphysics and complex hybrid models. It also considers the role of data-model fusion and stochastic effects in these models. Finally, it discusses the decomposition of complex systems into systems of canonical subsystems and the modeling of their behavior.

Advances in modeling complex systems will enable DOE to answer questions such as:

- Can we predict the operating characteristics of a clean coal power plant?

- Can we modify chemical pathways in a plant to produce biodiesel?

- How do impurities affect the performance of a membrane in a hydrogen fuel cell?

- What are the performance characteristics of possible nuclear fuel sources for the next-generation nuclear reactor?

- What is the predicted land contact and force of an Atlantic hurricane?

While modeling and simulation of complex systems has provided enormous benefits throughout science and engineering within DOE, there is growing evidence that new developments in this discipline could have profound effects on scientific discovery and on technologies in diverse fields of applied science. Indeed, with mathematical advances in the areas discussed below, new modeling and simulation methods could revolutionize the way science and engineering is done, complement traditional observational science, enrich or displace traditional experiments, and expand the vistas of

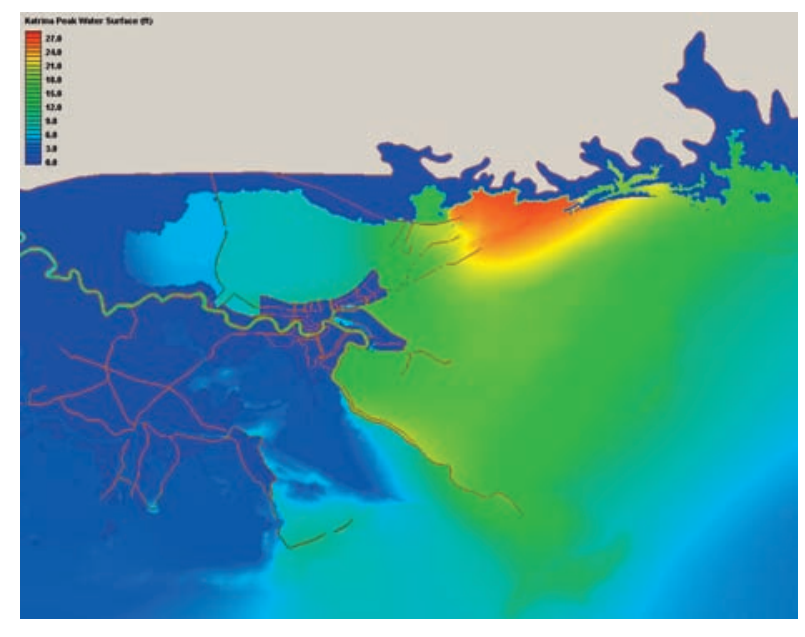

FIGURE 5. In the wake of Hurricane Katrina, U.S. Army Corps of Engineers used supercomputers at D0E's National Energy Research Scientific Computing Center to simulate storm surges as a step toward improving protective levees. The Overview simulation shows elevated storm surges along the Gulf Coast, while the simulation detail shows highest surge elevation (in red) striking Biloxi, Miss. New Orleans is the dark blue crescent to the lower left of Biloxi. Source: U.S. Army Corps of Engineers. 
scientific discovery beyond that otherwise achievable. We shall describe several areas in which fundamental research in applied mathematics can be leveraged to lift modeling and simulation to a new level and to equip many ongoing DOE programs with powerful tools critical to achieving their mission.

\subsubsection{Multiscale, multiphysics, and complex hybrid models}

Develop analytical and computational approaches needed to understand and model the behavior of complex, multiphysics and multiscale phenomena.

In traditional science, our knowledge of the physical universe is generally partitioned into specific scales, spatial and temporal, from parsecs and millennia representing the cosmos to angstroms and femtoseconds measuring events at atomic levels. The science of today and the future, however, must cope with events that transcend many scales. In most cases, we are lacking the mathematical foundations to make this transition. This "tyranny of scales," as some call it, is arguably the most important and difficult area of research facing advances in modeling and simulation. This relatively new field of research is referred to as multiscale modeling $16,17,18$. It attempts to develop broad theories of physical behavior that connect events at many levels, and has become a key issue in many important applications, including the design and analysis of advanced materials, nano-manufacturing, biological systems, drug design and delivery, and environmental models.

A parallel area of research of increasing importance is multiphysics modeling and simulation. Today's problems, unlike traditional science and engineering, do not involve physical processes covered by a single traditional discipline of physics or the associated mathematics. Complex systems encountered in virtually all applications of interest to DOE involve many distinct physical processes. For example, a complete computational model of a large-scale fusion device is a complex system involving issues of fluid dynamics, deformation of solid materials, thermal effects, ablation, fracture, corrosion and aging of materials, radiation and many other phenomena. All of these multiscale events must be properly modeled and interconnected for a viable predictive model of the behavior of the entire system. This requires new mathematical

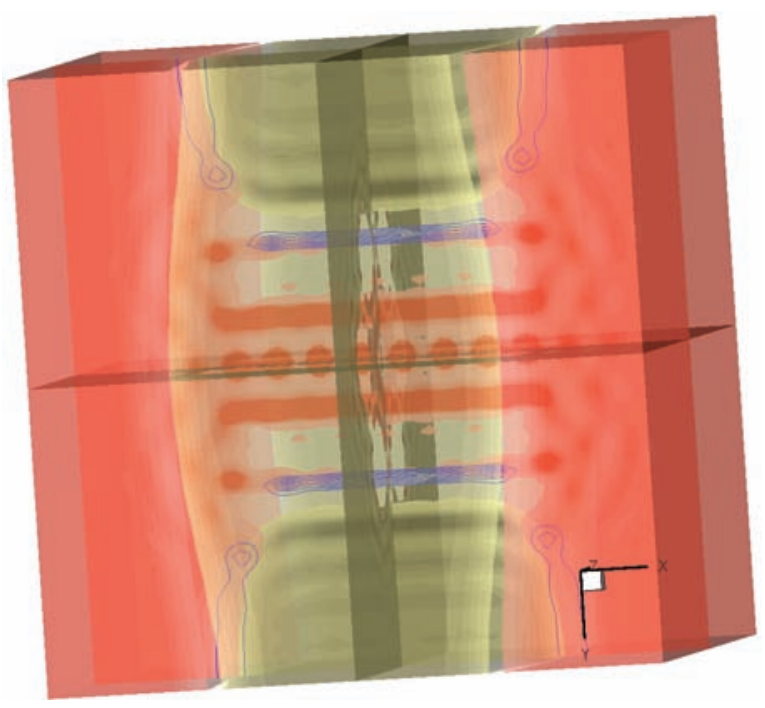

FIGURE 6. The Tramonto code was used to calculate the structure of a fluid bilayer as well as the surrounding water in the vicinity of an assembly of antimicrobial peptides based on a coarse-grained molecular model. The figure, based on 3-dimensional fluids-density functional theory calculations, is remarkable because it captures four distinctly different types of fluid structure simultaneously. These Fluids-DFTs can be characterized as integral equations of finite range that are neither sparse nor dense matrix problems. The theories are solved with a specialized segregated Schur complement approach developed at Sandia National Laboratories (SNL) for this class of problems. The particular solution shown in the figure was obtained as a result of coupling the Fluids-DFT algorithms with LOCA (the Library of Continuation Algorithms - also developed at SNL). The calculations were performed on 256 processors of the Red Squall platform. Source: Sandia National Laboratories

methods, new algorithms, and perhaps most importantly, significant new reformulations of appropriate models.

The issue of coupling models of different events at different scales and governed by different physical laws is largely wide open and represents an enormously challenging area for future research. Many of these problems involve the intermingling of discrete models with continuum models. For example, discrete molecular and atomistic models may be developed to study phenomena in nanodevices, material defects, or particulate flows, but these must be coupled in some appropriate sense with macroscale models that depict the medium as a continuum. These hybrid discretecontinuum models, the multiscale events governing their behavior, and the different physical theories underlying the coupled components represent challenging but vital areas of research in applied mathematics. 


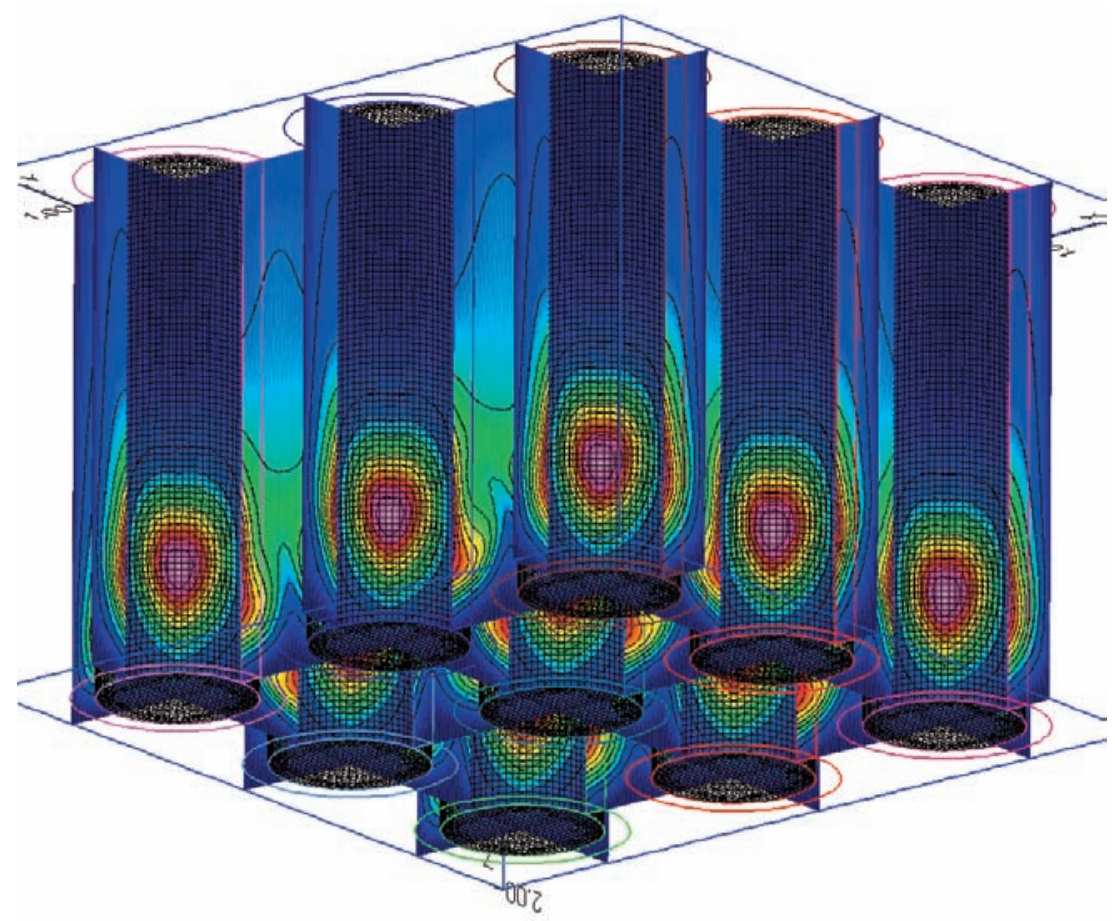

FIGURE 7. A multi-physics multi-domain computation of thermal-hydraulics conjugate-heat-transfer involving fluid flow past nine fuel rods in a simulated nuclear reactor The interior of each fuel rod is modeled as a solid with heat transfer and is coupled to an incompressible fluid that flows from bottom to top in the region around the rods. Source: Center for Applied Scientific Computing, Lawrence Livermore National Laboratory

Another source of complexity arises not from multiplicity of physical process or nonlinearities, but from the dimensionality of the problem.

Prototypical of this source of complexity are problems requiring the accurate modeling of quantum mechanical effects. Quantum mechanical systems have three spatial dimensions for each atomic particle in the system. Traditionally, assumptions are introduced at the level of the formulation to reduce this dimensionality. Mathematical techniques are needed to derive computational approximations that are appropriate when traditional assumptions are not applicable.

Specific strategies for meeting these challenges are to:

- Develop analytical tools for decomposing complex, multiphysics systems into their component processes and for elucidating the coupling between these component pieces;

- Develop methodologies for representing behavior at fine scales in models for the system at larger scales. Develop the corresponding analytical tools and computational approaches needed to quantify the impact of the fidelity of finer-scale models on large-scale dynamics;

- Develop algorithmic techniques appropriate for emerging computer architectures for simulating multiphysics and multiscale processes with quantifiable fidelity;

- Develop and analyze numerical methods for hybrid models that couple continuum and discrete processes. How do changes in the discrete variables affect the accuracy of the continuum part of the model?

- Develop approaches for deriving computationally tractable approximations to systems that are formulated in very high dimensional spaces, such as those arising in quantum mechanics.

\subsubsection{The role of data-model fusion in prediction}

Enhance the theory and tools for complex multiscale, multicomponent predictive models when observational or experimental data are incorporated in an essential way.

The fusion of observational and experimental data with advanced simulation promises to provide greater understanding of complex multicomponent multiscale systems than either approach alone can achieve. For example, experimental measurements are limited both in terms of the kind and frequency of observations that can be taken and may only provide access to limited aspects of a complex system. Predicting the long-term safety of storing 
radioactive waste at the Yucca Mountain repository provides an example of such a situation. We can perform detailed experiments to test the safety of waste containers, but can obtain only crude and indirect information about other components, e.g., porosity of the earth substrata in the region and frequency and duration of volcanic and seismic activity. Moreover, we can make direct measurements only over a relatively short time span in comparison to the time span for desired prediction, which is measured in tens of thousands of years.

There are three types of data-model fusion based on the relative dependence on data versus process model. First are applications where empirical predictive models of system behavior are based entirely on experimental data. These problems are frequently high-dimensional, complex and span both space and time, thus raising serious computational hurdles. Examples of these applications can be found in waste management and environmental remediation. The second are predictions based on simulations of physics-based mathematical models. While the predictions of system behavior are mainly based on knowledge of the physics, experimental data plays a key role in determining constitutive relations, in data and parameter values, and in the validation of prediction results. An example is provided by the challenges associated with the design of next-generation nuclear reactors, where both engineering design of the reactor and performance evaluation of potential nuclear fuels is required. The third are applications that require a combination of experimental and model simulation known as data assimilation. In this approach, observations of the current and past states of a system are incorporated into the simulation regularly with the objective of producing a data-informed analysis that can predict states outside the observation range. This approach has been used successfully to improve weather forecasts, and has potential broad applicability to many DOE applications.

The general state of the art in theory and computational methodology for data-model fusion is woefully underdeveloped, despite significant successes in particular applications. Although there are many approaches, as yet there is no standard software. What is needed are rigorous mathematical frameworks and efficient, robust implementations for data assimilation, including the development of suitable metrics for comparing model output to system observation. New approaches that move past the standard methodology, e.g., based on Bayesian formulations using Gaussian process models, are required. Given the expense associated with model solution, approaches that minimize the number of simulation calculations must be developed. There are serious implementation issues that must be addressed, such as storage requirements for variational approaches, advanced code generation based on automatic differentiation, and optimal control of computational errors in data-model fusion.

Specific strategies for meeting the challenges presented by data-model fusion are to:

- Develop systematic mathematical approaches for constructing nonlinear empirical models informed by physics principles, possibly including physically imposed constraints;

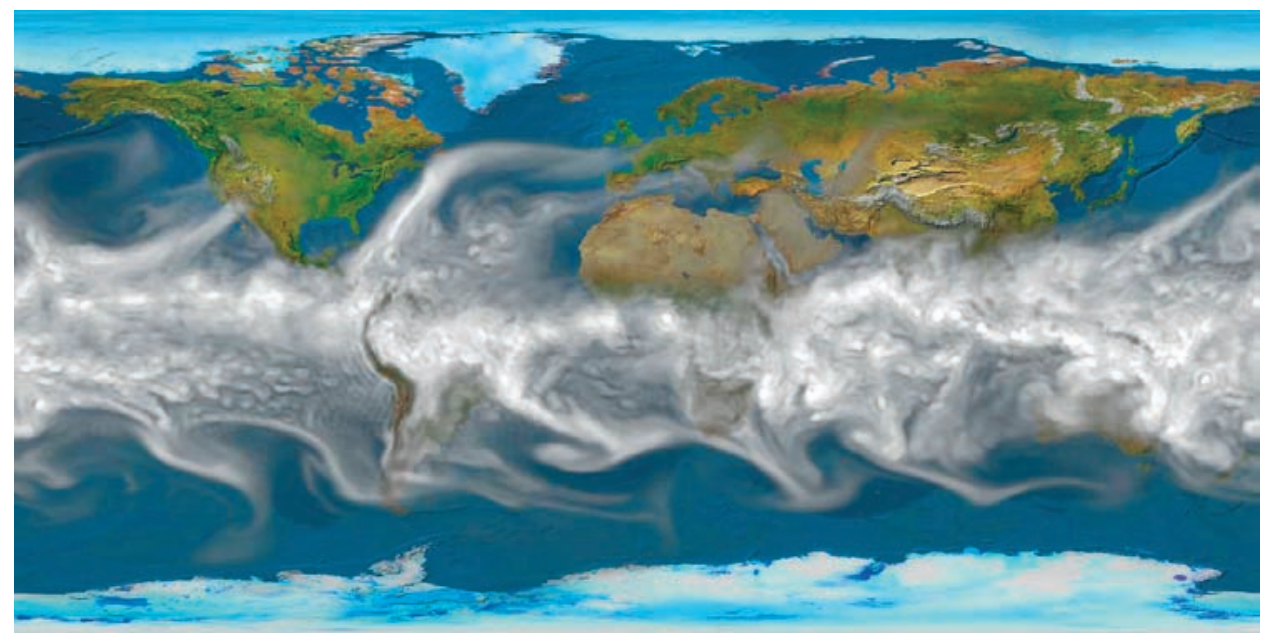

FIGURE 8. Increased computing power and improved algorithms enable scientists to see finer details in climate simulations. From the atmospheric component of the Community Climate Simulation Model, this visualization shows precipitate water from a high-resolution experimental simulation (T170 resolution, about $70 \mathrm{~km})$. Source: SciDAC Earth System Grid 
- Develop systematic methodologies for the estimation of system parameters, constitutive relations and uncertainties based on data;

- Develop mathematically rigorous frameworks and efficient, robust numerical methods for data assimilation into models of complex systems that are informed by numerical analysisbased error estimates for simulations and statistics-based error estimates for the assimilated data.

\subsubsection{Modeling stochastic effects in complex systems}

Develop new approaches for efficient modeling of large stochastic systems.

The random nature of events that occur in the physical world and throughout our everyday experiences have been well recognized for more than a century and a rich mathematical foundation has been developed for studying classical stochastic systems. Recent advances in engineering and science enabling manipulations at the microscopic scale to drive processes at the macroscale have raised a number of problems in which modeling of discrete stochastic and multiscale systems is a central issue. For example, probabilistic or stochastic approaches must be employed in physical situations where the number of molecules involved is too small for the continuum hypothesis to hold, yet full deterministic information is also not available or is inappropriate to describe individual molecular trajectories and collisions. Examples of stochastic behavior arise in the study of physics of rarified gases such as those in fusion experiments where long mean free paths imply that fluctuation effects become important, biological reactions where there are only a few molecules available to interact so that traditional (e.g. Arrhenius) reaction kinetics cannot be employed, and in general for models that describe the coupling of molecular physics into meso-scale or continuum scale equations. The increasing speed and power of computers has enabled stochastic simulation of some relatively simple models, for example, in molecular biology. However, the simulation of more complex and spatially dependent stochastic and multiscale systems will require new mathematics to justify the necessary approximations.

Another recent development has arisen with the realization that the predictability of large-scale models of complex systems must correctly take into account the uncertainty that results from experimental errors and the random nature of the data defining the system. For example, in biology, even cells of the same type have differences-they may be bigger or smaller, younger or older, etc., and some system parameters can be very difficult to measure and hence are subject to large uncertainties. Uncertainties in data must be quantified and propagated throughout the system to produce results with quantifiable levels of uncertainty. The uncertainties can take the form of errors and/or variations in the data, or of stochastic perturbations to the model due to external noise. We will say more about this huge and complex problem later in our discussion of the role of data and of the quantification of uncertainty. The fundamental importance of accounting for the variability of data in computer predictions is transforming how simulation is done and presents many open issues in the mathematics of stochastic systems.

Among these questions are those concerned with adding spatial dependency to the simulation of discrete stochastic and multiscale systems. The additional compleixty is analogous to that involved in the transition from solving ordinary differential equations to solving partial differential equationsin continuous deterministic simulation-the computational complexity increases by several orders of magnitude, in addition to some very challenging physical and algorithmic issues. Both new mathematics and the efficient use of advanced computer architectures will be required to tackle these problems. The field of mathematical modeling of largescale stochastic systems is, in many respects, in its infancy. This area is, therefore, a very important one for applied mathematics research and will impact many problems of interest to DOE.

Specific strategies in this area are to:

- Develop fast methods for discrete stochastic simulation that can effectively utilize next-generation computer architectures;

- Develop adaptive multiscale discrete stochastic simulation methods that are justified by theory and which can automatically partition the system into components at different scales;

- Develop new algorithmic approaches for large stochastic systems, particularly spatially dependent systems; 
${ }^{a}$ Develop efficient strategies for estimating the probabilities of rare events; particularly Monte Carlo approaches for sampling the tails of distributions;

- Develop the mathematical tools for sensitivity analysis, model development and optimization of large stochastic systems.

\subsubsection{Networks, systems and systems of systems}

Develop mathematical techniques for decomposing complex systems into systems of canonical subsystems and modeling their behavior.

A large number of complex systems that operate within the purview of DOE involve networks and heterogeneous assemblies of different system types, which collectively operate as a single system. Advances in simulation and modeling have enabled the solution of complex system-wide models, as well as models of different system components. The development of systems of systems (SOS) models, which integrate these disparate models into a single analytic framework, is a natural extension of this capability that promises to provide more detailed predictions of system-wide dynamics and interdependencies.

Motivating examples of SOS applications include modeling the national power grid, planning for investments in energy infrastructures, characterizing supply chain management in the nuclear power industry, and planning for the impact of new technologies on national security. These applications address large-scale inter-disciplinary problems involving multiple heterogeneous, distributed systems that are embedded in networks at multiple levels and in multiple domains. SOS models often need to be developed by integrating a variety of existing models, and these models may need to be developed using expertise from different disciplines. Thus, SOS models often represent complex dynamics that reflect a lack of centralized policymaking and control.

The effective size and complexity of SOS models will require a new paradigm in modeling and simulation. Simple integration of existing modeling capabilities is not likely to enable the modeling of large-scale systems. Integration of models with different temporal and geographic scales poses many of the same challenges seen in multiscale physical simulation models. Further, integrating different modeling and simulation techniques remains an outstanding challenge in many applications. For example, discrete network models can effectively model interactions amongst subsystems, but these models need to be effectively integrated with detailed models of subsystem dynamics. Finally, the computational cost across SOS sub-models needs to be managed to enable effective scalability to national-scale applications.

Another key challenge for SOS models is characterizing their dynamics and optimizing their performance. Validation of SOS models is a critical challenge for their use in practice, and this is hampered by the fact that it is difficult to predict the dynamics of complex SOS models containing heterogeneous subsystems. In practice, the analysis of so-called emergent behavior in these models will be critical for model validation, and for the assessment of the robustness of SOS dynamics. Further, there remain many challenges when considering the optimization of SOS models to improve performance, as well as the analysis of SOS dynamics in extreme conditions. Such analyses are particularly challenging due to the computational cost of SOS simulation models, and thus parallelization of SOS frameworks is an important technical goal.

Specific strategies in this area are to:

- Develop mathematical approaches for modeling the behavior of hierarchical networks in which subnets are controlled autonomously. For example, develop mathematical approaches for analysis of networks of intelligent agents;

- Develop techniques for decomposing complex systems into canonical subsystems.

Characterize canonical system interfaces that support scalable SOS models;

- Develop parallelization schemes for SOS models. What parallel architectures can effectively simulate these models? How can SOS models exploit emerging computer architectures?

- Developing modeling approaches for using large-scale network system models with detailed models of subsystems;

- Develop analysis methodologies for discovering and characterizing emergent SOS behavior. How can visualization and data analysis be used to aid the modeler in the discovery of unexpected dynamics? 
- Develop Monte Carlo methodologies for rapidly exploring worst-case scenarios in complex systems.

\subsection{Analyzing the behavior of complex systems}

Address the challenges of analyzing and understanding the behavior of mathematical models for complex scientific and engineering systems.

The development of models for complex systems is just one step in the process of developing scientific understanding. Techniques are also needed for analyzing the behavior of these systems. The analysis and understanding of complex systems will enable questions important for the DOE mission to be addressed, such as

- How stable is the plasma containment in a Tokomak?

- Can we safely sequester $\mathrm{CO}_{2}$ underground?

- How will changes in the demand for electric power affect the stability of the power grid?

- How quickly is climate change in the terrestrial system occurring and what is the uncertainty in the predicted time scales? What are the greatest sources of climate change and how can society most effectively reduce the rate of change?

- What is the level of safety of storing radioactive waste at Yucca Mountain? What are the possible consequences of failures in any component of the storage facility?
- How quickly can an introduced bio-weapon contaminate the agricultural environment in the United States?

Research in sensitivity analysis, uncertainty quantification and in the role of data in the analysis and understanding of complex systems is essential for addressing these and a host of similarly complex questions for DOE.

\subsubsection{The role of data in the analysis and understanding of complex systems}

Develop sound, computationally feasible strategies and methods for the collection, organization, statistical analysis and use of data associated with complex systems.

The ability to understand the behavior and predictive capability of models for a complex system depends in an essential way on the data associated with that system. Such data comes in many forms-observational data of varying quality, multiple types of experimental data and the results of computational simulations. The researcher who uses and analyzes this data also faces significant challenges:

- The data may be voluminous, yet provide only a sparse representation of a complex system, e.g. in the common case of a high dimensional problem;

- Data is frequently both spatially and temporally heterogeneous;

- Data from experimental measurement or simple observation is rarely presented in a form

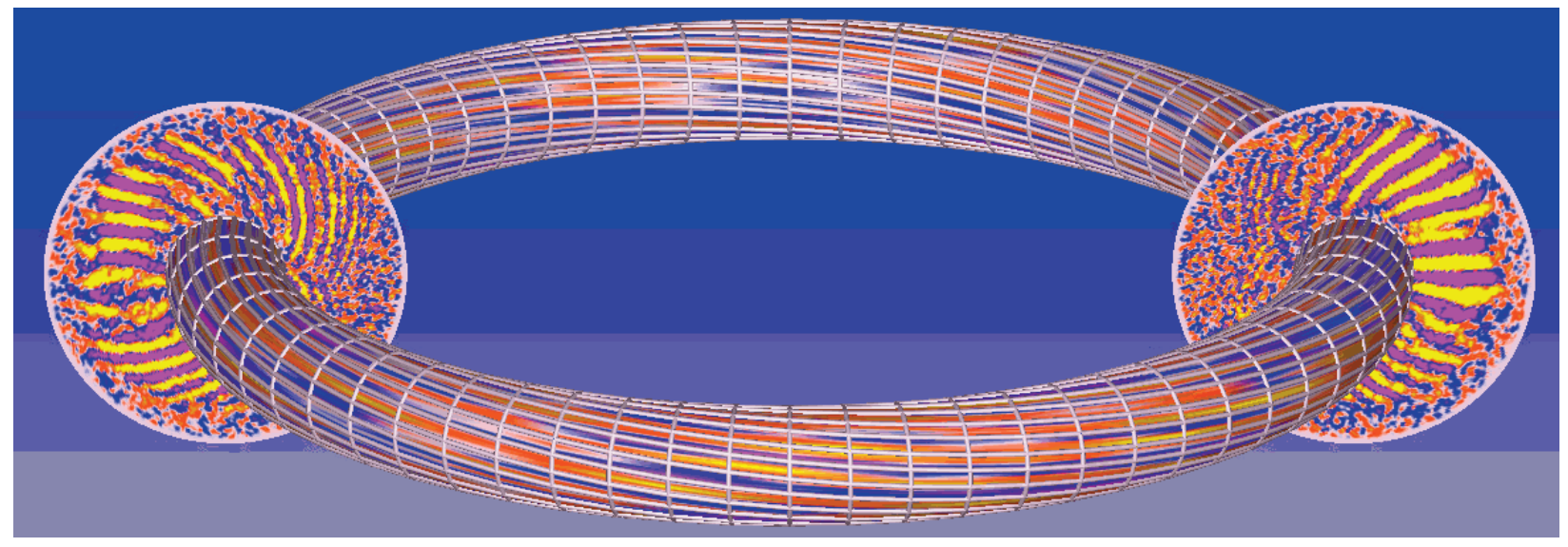

FIGURE 9. Multiphysics simulations will be required to develop a complete understanding of future magnetic fusion devices. This figure shows potential contours of microturbulence for a magnetically confined plasma. The finger-like perturbations (streamers) stretch along the weak field side of the poloidal plane as they follow the magnetic field lines around the torus. Source: Princeton Plasma Physics Laboratory. 
that is immediately practical for mathematical modeling;

- The collection, storage and processing of data often entails tremendous cost, Therefore, careful consideration must be given to what data to collect, particularly given the vast choices of data collection to support the development and use of complex systems.

Mathematics will play an essential role in addressing these challenges, providing rigorously justified techniques for data representation and transformation, the combining of different types of data, and in the development of effective data collection approaches, particularly in the face of constrained resources.

Different statistical representations of data are useful for different kinds of analyses and mathematically well-founded techniques for transforming data are crucial. For example, transforming information between different spatial or temporal scales may need to be accomplished when only sparse data are available to support a high-resolution simulation, such as in simulations of subsurface porous media flow. Statistical and mathematical analysis of large, heterogeneous data sets is a daunting challenge, raising the need to develop new mathematical approaches for dimensional reduction in order to discover the essential features represented in the data. Non-linear principal components analysis, topological data analysis techniques, support vector machine approaches and functional data analysis are examples of dimensional reduction approaches that might be developed further.

In many cases, researchers are faced with many different data sets of incongruent size, quality and type. For example, understanding the performance of a nuclear weapon requires the melding of data from historical full systems tests, small-scale experiments and integrated experiments with complex physics simulations. Similar challenges abound in climate analysis, material design, accelerator design and combustion modeling. Bayesian, network, graphical and hierarchical models are beginning to emerge to address the challenges in combining heterogeneous data types in space and time.

Faced with an explosion of observational and simulation technology that offers many options for data collection and generation, often in huge quantities, researchers must answer the question of how

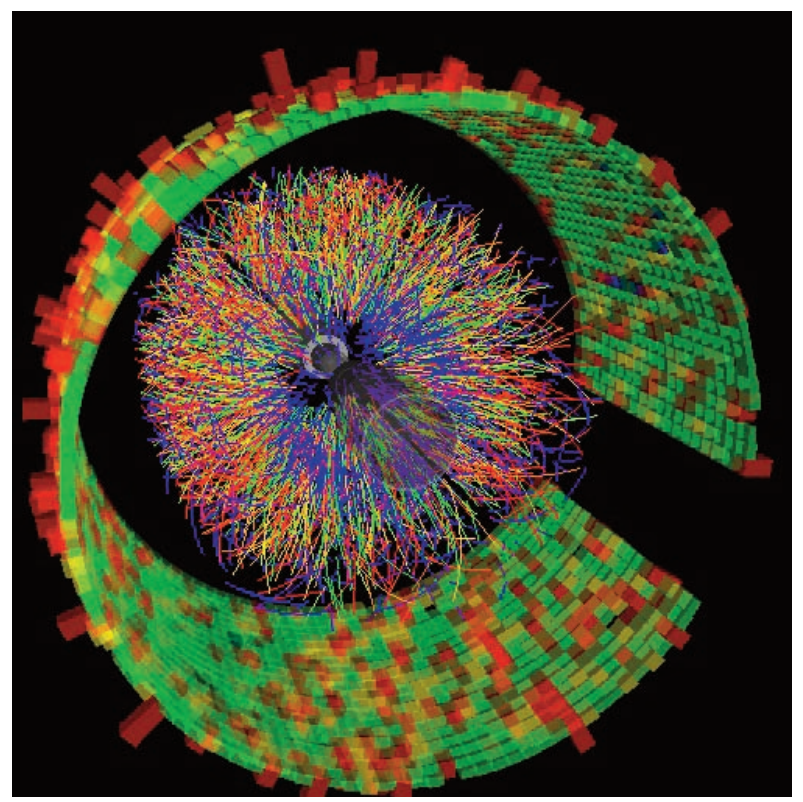

FIGURE 10. The STAR Experiment at Brookhaven National Laboratory generates petabytes of data resulting from the collisions of millions of particles. Data management tools make it easier for scientists to transfer, search and analyze the data from such large-scale experiments.

to design experiments, both physical and computational, to optimally collect data. The traditional design of experiments is concerned with allocating trials within a single, typically physical, experiment. As computation has emerged as an experimental tool, new methods have been developed to optimize data collected from computational experiments as well. To tackle the complex multiscale, multicomponent systems that DOE needs to understand in the future, mathematics must be developed that will enable researchers to move well beyond the design of physical and computational experiments to the design of hybrid methods that can simultaneously optimize data collection from across a wide range of information types, including multiple types of physical experiments, computational experiments, historical records and expert judgment.

Specific strategies for meeting these challenges are to:

- Develop efficient methods for the statistical analysis of large, heterogeneous data sets;

- Develop rigorous mathematical and computationally feasible approaches for combining data of different types and of different quality that 
can also quantify the various forms of uncertainties in the data;

- Develop rigorous but computationally feasible methods for dimensional reduction of data;

- Develop hybrid experimental design methods to optimize the collection of observational, experimental, computational and historical data for complex systems.

\subsubsection{Sensitivity analysis.}

Advance the theory and tools for sensitivity analysis to address the challenges posed by complex multiscale, multicomponent models.

Sensitivity analysis is the organized study of the way in which the output of a model responds to variations in model inputs (parameters, initial and boundary conditions) and in the model itself.

Inputs into a model are subject to many sources of uncertainty, variability and measurement error.

The model itself may be subject to uncertainty arising from incomplete information or poor understanding of the physical processes and driving forces. The coupling of different physical processes in a model through a range of scales, where variability and uncertainty in one physical component affects the other components, also affects the output of the model. True predictive simulation requires an understanding of how all of these issues contribute to the uncertainty in observed model outputs.

Sensitivity analysis provides a methodology for quantifying the stability of model output with respect to given changes in parameters and initial and boundary conditions. This information can be compared to how the physical system depends on the conditions represented by physical data. Sensitivity analysis is also a powerful tool for investigating which components of a model contribute the most to variability in model output and hence must be better represented computationally. Likewise, sensitivity analysis can be used to determine regions of the parameter space and initial and boundary conditions for which the model output variation is most significant and to find regions in parameter space that lead to significant changes in the physical behavior of the model, e.g., bifurcation points.

Inverse sensitivity analysis reverses the point of view, and is used to determine the allowable uncertainty in inputs to a model given a desired degree of uncertainty in the model output. This can provide a powerful tool for linking model results to experimental data. Coupled with experimental design, this information can then be used to determine which parameter and data values need to be measured most accurately in an experiment. When predicting the behavior of a complex system, e.g., a climate model that depends on hundreds of parameters that are difficult to determine and expensive to evaluate, any information on the relative importance of parameters and the requirements for their accurate determination can lead to effective prioritization of experiments and data collection.

When considering sensitivity analysis of complex multiscale, multicomponent systems, additional theoretical hurdles arise. There are no general methodologies for treating the effects of variation and uncertainty in high dimensional parameter spaces that include strong interdependencies between parameters and large-scale ranges in parameter values. A large parameter set often leads to a wide range of model behavior, particularly if bifurcation point behavior occurs, making the interpretation of sensitivity analysis results difficult. There are few good methods for understanding the covariance structures of observational data versus that of model predictions. Sensitivity analysis of a complex system typically benefits from a fusion of mathematical and statistical techniques, yet mathematical frameworks for combining such approaches in a unified fashion do not yet exist.

These characteristics also mean that sensitivity analysis of a complex system is very computationally intensive. Well-established experimental design and analysis techniques for studying the input-output spaces for computational models typically fail as models become more complex. Methods for statistical design of efficient and accurate sampling procedures typically do not take full advantage of the implicit mathematical structure of complex models. The large computational overhead associated with existing methods of sensitivity analysis is a significant impediment to their use. Approaches that exploit mathematical structure or that effectively take advantage of characteristics of high-end computers can typically require invasive changes in simulation codes, which can be undesirable when dealing with large legacy codes in production environments. 
Not least of the difficulties associated with sensitivity analysis of complex systems is a need for efficient methods to organize, visualize and understand sensitivity analysis results. Large-scale ranges, highdimensional parameter spaces, and high-dimensional output from analysis all conspire to make the interpretation of analysis results very difficult.

Specific strategies for meeting the challenges of sensitivity analysis for complex systems are to:

- Develop accurate, efficient computational tools for sensitivity analysis and inverse sensitivity analysis of complex systems characterized by high dimensional parameter and data spaces and wide ranges of model behavior;

- Develop mathematical and computational frameworks for fusing a variety of statistical and deterministic analysis approaches for sensitivity analysis, for inverse sensitivity analysis and for model calibration;

- Develop efficient and effective methodologies for presenting and interpreting the results of sensitivity analysis of complex systems.

\subsubsection{Uncertainty quantification and mitigation.} Significantly advance the theory and tools for quantifying the effects of uncertainty and numerical simulation error on predictions using complex multiscale, multicomponent models and when fitting complex models to observations.

While sensitivity analysis seeks to relate the outputs of a model to its inputs, uncertainty quantification casts a much broader net in terms of assessing confidence of predictions based on all available information. Predictive uncertainty is associated with the combined effects of limitations in sensitivity and accuracy of physical measurements, incomplete understanding of the underlying physical processes, the complexity of coupling different physical processes across large-scale differences, and the numerical errors associated with simulations of complex models.

The quantitative understanding of predictive uncertainty is essential when predictions are to be used to inform policy making or mitigation solutions where significant resources are at stake. For example, an understanding of predictive uncertainty played an essential role in the acceptance of the need to design policies to address global warming where the cost of different choices varies by trillions of dollars.
There are a number of significant challenges associated with uncertainty quantification and mitigation for complex multiphysics systems. Uncertainty comes from many sources, e.g., from experiment and computation; it can be represented in different ways mathematically, e.g., statistical, probabilistic, and deterministic; and is even associated with situations where it is unclear how to mathematically represent that uncertainty. In spite of the many approaches for representing uncertainty, it is important that mathematically consistent approaches for relating various types of uncertainty be developed.

Not only can uncertainty enter into a complex model through a number of avenues, the subsequent propagation of uncertainty through the model is also a complex process. New analytic and computational methods must be developed for quantifying the effects of uncertainty and numerical error on model predictions, model calibration, and data assimilation analysis based on what is known about the uncertainty in inputs and the accuracy of numerical methods. Because multiscale, multiphysics simulations are often computationally resource-limited, uncertainty analysis methods that are robust with respect to limited numerical accuracy must be developed.

Uncertainty quantification is also costly in computational terms and often requires intrusive additions to existing software. There is a strong need for both theoretical and computational approaches for adaptive sampling and numerical solution that lead to robust and efficient control of uncertainty when possible.

Specific strategies to support uncertainty quantification and mitigation are to:

- Develop mathematical, statistical and hybrid approaches for describing and combining uncertainty and error from multiple sources presented with multiple representations;

- Develop mathematical, statistical and hybrid approaches for analyzing and quantifying the effects of uncertainty and error propagated through a complex model on model predictions, model calibration and data assimilation analysis;

- Develop memory-access-efficient algorithms that match current and emerging computer architectures, reducing or eliminating the need for multiple runs in sampling-based approaches. 


\subsection{Using complex systems to inform policy-making}

Develop the mathematics needed to inform policy-makers based on the prediction, optimization and understanding of complex natural and engineered systems

Advances in mathematical modeling provide ever-expanding capabilities for the prediction and understanding of the behavior of complex systems, both natural and engineered. The next step is answering the myriad questions that arise in policymaking involving these systems, such as:

- How can new materials be designed to display a specified list of desirable properties, and what are the tradeoffs among these properties?

- What is the optimal design of a MEMS-scale electromechanical device?

- Which characterization of equilibrium constraints is most suitable for designing a compact stellarator experiment?

- What are the optimal locations and communication protocols for sensing devices in a remote-sensing network?

- What changes should be made in models of the atmosphere and clouds to most effectively incorporate newly collected data for improved climate change predictions?

- Which statutory constraints on operating procedures have the greatest influence on power plant efficiency?

- What are the most harmful health-related outcomes of exposure to nuclear waste in the short term?

- How quickly could the United States recover if part of the power grid became inoperable?

- What are the most likely effects if a future energy technology fails to live up to its current expectations and timeline?

Research in risk analysis, optimization and inverse problems is crucial for addressing these and a host of similarly complicated questions for DOE.

\subsubsection{Risk analysis}

Significantly advance the mathematics that supports risk analysis techniques for policy-making about complex systems that include natural and engineered components, and economic, security and policy consequences.

Risk analysis-broadly defined as the provision of rigorous procedures to evaluate sources of risk and their consequences-is an integral part of policy-making in activities with potentially negative outcomes. Risk analysis is ubiquitous in business and finance, where the negative consequences tend to be financial, and in gauging the health risks of drug development and medical trials. DOE has responsibilities that if not executed safely and correctly bear awesome potential for harm, potentially resulting in nuclear accidents, toxic and radioactive waste releases, power grid failures, oil spills and groundwater contamination. In addition, DOE must undertake high-stakes policy-making with unavoidably incomplete information about related issues of science, engineering, logistics and security.

Examples of this are the uncertainties associated with safe transportation and storage of nuclear waste at Yucca Mountain, or in the simultaneous evaluation of the safety, surety and reliability of the nuclear weapons stockpile.

Traditional risk analysis is based on mostly well-defined static objectives and constraints, but these are woefully inadequate for most DOE problems, especially those that depend on incorporating scientific insights from complex nonlinear models. Significant mathematical advances will be required to more effectively address the challenges of policy making supported by models of complex systems.

To address DOE's needs, mathematics research supporting risk analysis is required in several areas:

- Combining multiple sources of information, including theoretical models, test and observational data, computer simulations, and expert knowledge from scientists, field personnel and policy makers;

- Developing formal methods to integrate disparate information sources relative to the contents of the evaluation (for example, simultaneous assessment of performance, reliability, sustainability, dependability and safety);

- Addressing the varied needs of multiple stakeholders in policy making involving, for example, complex resource allocation (e.g., in mounting a full system test or building a new experimental facility) or continuous evaluation (e.g., support- 
ing decisions that need to be made at multiple times and at unpredictable intervals);

- Incorporating explicit uncertainty and dynamic changes in the evolution of knowledge about the system being studied.

Research in support of analysis and assessment of risk for highly complex systems, in the face of uncertainty, requires a unified approach founded on several disciplines, including statistics, probability, computer science, decision theory, graph theory, knowledge elicitation and representation, not to mention modeling and simulation. Only through the language and theory of mathematics will representations and analyses for risk assessment be developed that can bridge these disciplines.

Effective analysis of the complex risks that DOE faces in the future demand a much broader approach than is taken today for knowledge elicitation, representation and transformation. Ontologies, which are the models that represent concepts and relationships from differing "cultures" such as science, economics, security and public policy, must be formalized to so that problems in overlapping domains can be addressed using methods that are both expressed in the various "native languages" of the collaborating experts and are mathematically tractable. This process involves iterative cycles of representational refinement and quantification, resulting in predictive statistical models that make intuitive sense to all parties. The need for better ways to represent knowledge and assess risk is especially urgent because of DOE's increasing reliance on multidisciplinary teams in which mathematicians are expected to develop predictive models integrating multiple types of data, information and knowledge.

The way in which information is organized also has a major influence on how that information is used. With the wide range of information (and knowledge) needed for DOE's continuous policymaking responsibilities, it is critical that this information is formally organized. This will require a variety of tools to capture information, organize it and make the results available to different communities, including policymakers and the public.

Incorporating the results of complex mathematical and computational models into risk analysis is extremely challenging because of unresolved issues about calibration and validation of models based on limited experimental and simulation data. This is especially true when the model outputs are high dimensional. Additionally, the need to systematically include uncertainty and risk when developing the models initially takes mathematician/statisticians outside the "comfort zone" of traditional probability theory and into uncharted areas of fuzzy measurement, belief functions and possibility theory. Approaches that expand Bayesian inference, Bayesian hierarchical models and techniques from spatial statistics to risk assessment grounded in

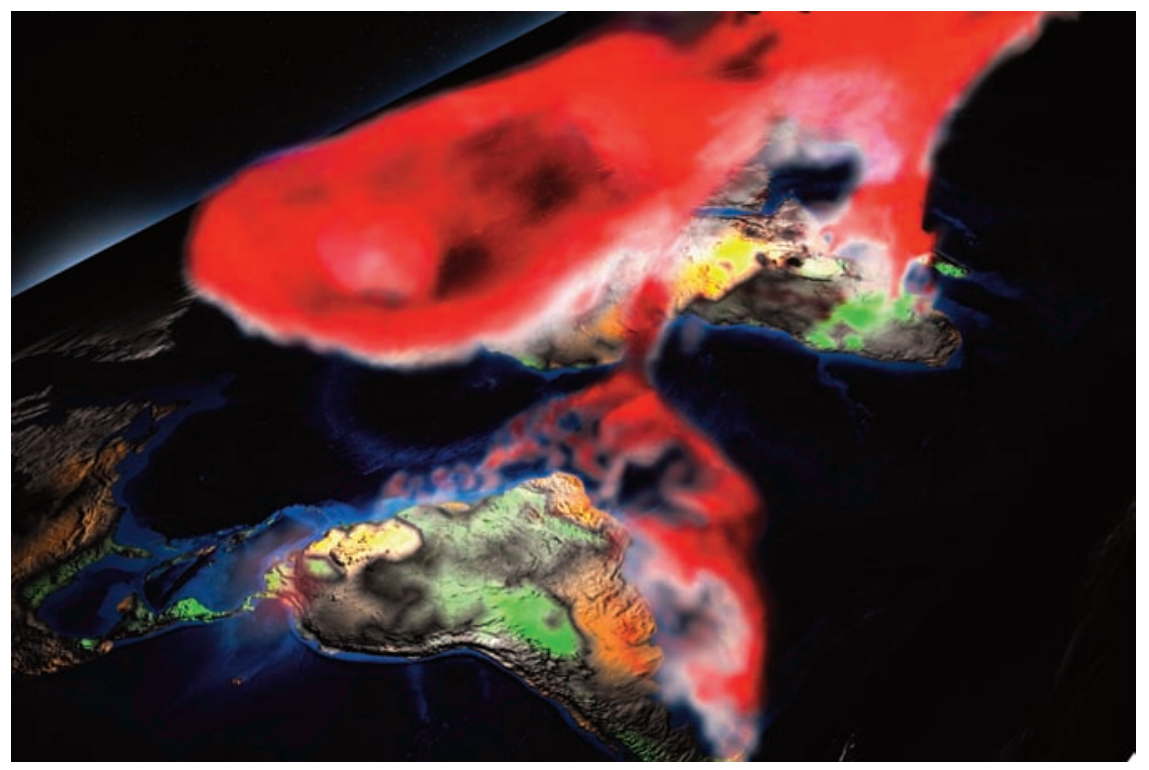

FIGURE 11. Computation is an important element in developing an understanding of global climate change. This image shows a snapshot of the simulated time evolution of atmospheric carbon dioxide (the red plumes) concentration originating from the land surface at the beginning of the industrial carbon cycle (around 1900). This CO2 is a product of the net ecosystem exchange, the CO2 flux due to respiration of vegetation and soil microbes (green areas on land) minus that taken up for ecosystem production (orange areas on land). The underlying simulation is one of a number of runs performed for Phase 1 of the Coupled Climate/Carbon Cycle Model Intercomparison Project. Source: National Center for Atmospheric Research; Oak Ridge National Laboratory 
complex models will be required to address these challenges.

Strategies for meeting these challenges are to:

- Develop mathematically rigorous conceptual graphs and statistical graphical models for translating from qualitative to quantitative representations;

- Develop mathematically based risk assessment techniques whose reliability can be estimated accurately-even when minimal data is available for the system being studied;

- Expand Bayesian inference, Bayesian hierarchical models and techniques from spatial statistics to risk assessment grounded in complex and frequently computational models;

- Develop reliable and mathematically sound techniques for deducing information from data and knowledge sources that are high dimensional and heterogeneous in nature and quality;

- Perform research in knowledge management to support risk analysis with a focus on the development of analysis tools that can be accessed and used by a wide range of interested parties.

\subsubsection{Optimization}

Develop techniques for formulating, analyzing and solving challenging optimization problems arising in complex natural and engineered systems.

Optimization is a broad and pervasive paradigm for determining and characterizing the "best" results. The field has made great progress for more than 40 years, and a wide variety of optimization problems once viewed as unsolvable are regarded today as routine. Nonetheless, further research in optimization is essential because many of DOE's important policy-making decisions involve optimization problems that are either beyond the capabilities of today's state of the art or else provably intractable in their most general form.

DOE applications increasingly result in nonlinear optimization problems with a mixture of variable types. For example, the design of fossil energy power generation systems involves both continuous quantities such as length and width, and integer variables such as the number of processing units. Categorical variables (those that lack a natural ordering, such as alternative energy technologies) are also common in policy-based applications, and occur, for example, in determining the optimal loca- tions for placing sensors or designing nanomaterials. Since there are no guaranteed solution techniques for general nonlinear mixed-variable problems, progress depends on developing theory and algorithmic strategies that exploit special structure. These might include fast algorithms that approximate the solution within a guaranteed factor of optimality, or techniques that use randomization to produce an accurate solution with very high probability.

The most-studied objective functions in optimization problems are assumed to display some degree of smoothness, yet non-smooth behavior is common in the real world-for example, the collapse of an aging material or the splitting of a single flow path into two. Similarly, constraints are often described as equalities or inequalities involving a continuous function of the variables, but nonsmooth constraints, including equilibrium, complementarity and disjunctive constraints, also appear prominently in DOE applications such as computing electric power market equilibria. Several sophisticated techniques have been developed for handling problems whose constraints are all of a single type, but theory and algorithms are lacking for problems with a mixture of constraint forms.

The basic formulation of an optimization problem can also significantly affect its mathematical and computational tractability. Two problem classes that remain challenging for today's state of the art are multilevel and multi-objective optimization. In multilevel or hierarchical optimization, there is an explicit stratification from highest to lowest in which each level solves its own optimization problem, dependent on the solution of problems from higher levels. This multilevel optimization occurs, for example, in DOE energy models that include federal and state governments, where federal regulations impose fundamental constraints on the locally determined objectives of each state. Multiobjective optimization occurs when several objectives, typically conflicting, are to be optimized simultaneously-for example, in finding an energy source that is both inexpensive and non-polluting. Multilevel and multi-objective optimization problems are extremely difficult even for the simplest linear models, becoming "impossible" as the dimension increases and nonlinearities become more prominent. Research is therefore necessary to formulate and solve specially structured versions of 
these problems in which the number of variables and the degree of nonlinearity are realistic.

Some DOE problems, notably in biology and physics, are posed as the global minimization of a nonlinear function subject to constraints, and are solved using computationally intensive techniques based on physical intuition. Because of widely acknowledged inefficiencies in these approaches, even small advances in global optimization would be extremely valuable, including new results that mathematically characterize the efficiency and reliability of popular heuristics.

Very large-scale optimization problems can usually be solved efficiently only by taking advantage of known special structure of the underlying problem, e.g. by recognizing that the power grid is a sparse network of heterogeneous elements. Such problem characteristics often translate into identifiable matrix properties and can also be reflected in the symbolic representations deduced by a modeling language. Research on the distinctive matrix computation needs of optimization would greatly enhance solution speed for DOE's portfolio of extremely large problems with few, mild, or separable nonlinearities.

In science- and engineering-based contexts, especially in longstanding physics applications, scientists typically use techniques that are highly problem-specific, e.g., adjusting selected accelerator design parameters based on expert knowledge. Although often useful, this kind of approach can lead to inefficiency when hardwired into algorithms and software that are used later for other problems. Research in modeling languages geared to optimization would increase both flexibility and adaptability by allowing a cleaner separation between formulation and solution techniques.

The models appearing in constrained optimization problems involve uncertainty from various sources, and it is essential to characterize the quantitative and qualitative effects of such uncertainty on an "optimal" solution. When the associated perturbations are small, strategies are known for analyzing worst- or average-case effects; when a probability distribution representing the behavior of the uncertainty is available, stochastic optimization can be applied to specialized problem classes such as linear programming. However, techniques are needed for applications in which the uncertainties are large, nonlinear, and possibly discontinu- ous, such as in achieving mandated safety levels in a nuclear power plant.

Strategies for meeting these challenges are to:

- Develop analysis and algorithms for optimization problems with continuous, discrete and categorical variables, and with non-smooth and/or nonstandard objective and constraint functions;

- Perform theoretical and computational investigations of general-purpose and specialized algorithms for important subclasses of optimization problems, including problems that are intractable in general but solvable in particular cases of interest to DOE;

- Develop methods for finding approximate solutions of global optimization problems and for finding global solutions of problems with special structure;

- Develop algorithms for solving specially structured versions of multilevel and multi-objective optimization problems;

- Develop algorithms for fast solution of the highly structured, not necessarily sparse, linear algebraic sub-problems that arise in optimization;

- Create sophisticated modeling languages that allow large structured optimization problems to be expressed in the natural language of the user;

- Invest in analysis and algorithms for stochastic optimization, addressing the effects of nonlinearities, special structures and nonstandard probability distributions.

- Develop new optimization methods and algorithms that match the needs for increased concurrency and tolerance of memory latency in current and emerging computer architectures.

\subsubsection{Inverse problems}

Develop techniques for addressing the mathematical and computational difficulties of inverse problems associated with complex systems.

Inverse problems arise in applications where essential elements of a mathematical model are poorly known or understood. In a typical inverse problem, these model parameters, which may include inaccessible material properties or unknown initial or boundary conditions, are defined by minimizing the discrepancy between experimental observations and the model's predic- 
tions. Inverse problems occur in numerous DOE applications, including studies of contaminant transport, crude oil recovery, climate prediction, astrophysics and diagnostic and detection procedures such as non-destructive evaluation, where waves are transmitted through an unknown media in order to identify inhomogeneities.

Unfortunately, inverse problems are typically ill-posed-that is, a small perturbation in the observed data can lead to a large, even discontinuous, change in the estimated parameters. As a result, sophisticated techniques /often called "regularization") are used to prioritize otherwise indistinguishable solutions. Insights for regularizing inverse problems are often derived from a detailed physical understanding or theoretical analysis of specific properties of the underlying mathematical operators. Since inverse problems from different domains vary significantly in mathematical structure, their successful solution is likely to require close collaborations between mathematicians and application experts. In addition, since high-quality observed data is critical in solving inverse problems, mathematical research would benefit from ties with the collection of experimental data.

Research is also needed on reliable and computable metrics that complement regularization by providing essential information such as sensitivities and adjoints. A serious limitation of several standard regularization schemes is their assumption of closeness to linearity, which is not valid for the complex systems associated with the most challenging DOE applications. Numerical linear algebra is a key ingredient because matrix decompositions help to reveal the underlying structure of ill-conditioning; however, the most powerful linear algebraic tools available today, such as the full singular value decomposition, become prohibitively expensive computationally for complex problems of the increasing sizes that are interesting to DOE. Computationally tractable alternatives are needed to address very large problems of this type.
The solutions of most inverse problems will inevitably be uncertain to some degree. Depending on mathematical structure, such uncertaintieseven when tiny-can cause inverse problems to be so ill-posed that available mathematical techniques cannot yield meaningful results. The causes of these unfavorable conditions may not be obvious in advance. They may reflect an inappropriate model formulation, a less-than-optimal choice of regularization, or (most ominously) an inherent mathematical difficulty. Research is needed on techniques for understanding the multiple roles and implications of uncertainty in inverse problems.

Strategies for meeting these challenges are to:

- Develop and extend analysis tools for inverse problems, especially approaches that do not depend on near-linearity;

- Develop large-scale linear algebraic techniques that reliably capture the structure of ill-posedness, allow its analysis and guide subsequent model reformulation;

- Enhance connections between mathematical research in inverse problems and expertise/ experiments in important application domains;

- Develop techniques for understanding the connections between uncertainty in mathematical structure and in the chosen model formulation;

- Develop mathematical techniques for reducing uncertainty and/or dimensionality by incorporation of prior conditions.

The Department of Energy's Applied Mathematics program supports research that produces the mathematical advances needed to address our nation's energy, environmental, and national security challenges. This report has already discussed technical areas in which mathematics research could make major contributions to these problems, and we now turn to strategies for effective program leadership. 


\section{Driving Innovation and Discovery in Applied Mathematics Through Effective Program Leadership}

\subsection{Encouraging and rewarding risk-taking in research}

A fundamental practical difficulty in building and sustaining a strong research program is the universally acknowledged fact that basic research does not lend itself to detailed or accurate predictions of what its results will be, or when they will occur. Even "transformational" discoveries seldom take place in a vacuum, but rather depend on a long sequence of individual advances, some of which are small. Since "one cannot cultivate only the fruit of the tree", a balanced portfolio is necessary for a healthy research enterprise. ${ }^{19}$ The optimal form of balance cannot be specified precisely, but an essential ingredient is that researchers should be encouraged to take risks-which, to be genuine, include the possibility of failure.

From a researcher's perspective, the research endeavor involves two forms of risk. Significant advances of the kind desired by both the researcher and the sponsoring program are more likely if the researcher feels free to take risks in devising new scientific approaches. However, if researchers believe that their funding requires the production of measurable results within a strict timetable, the danger of losing funding acts as a strong disincentive to risk-taking in research. The obvious conundrum that faces any research program supported by public funds, therefore, is fostering risk-taking in research while requiring accountability through measured results. An example of the associated difficulties arises in designing a process for regular reviews of research progress. A significant and important challenge is to balance the review necessary for quality control with the need to encourage risk-taking.

For the past sixty years, national and international discussions of research policy have emphasized that the timeframe for transformational advances may involve ten to fifteen years of concentrated effort. Hence imposing a much shorter time limit (for example, three years) on all project support will inherently discourage risk-taking. One strategy for improving the likelihood of break- throughs while retaining accountability is to reward investigators and programs with a proven track record of achieving the program's goals with continued long-term support. At the same time, it is also important to invigorate the flow of ideas-for example, through mechanisms to bring new researchers, especially those early in their careers, into the research program.

Finally, the program can support activities where a diverse group of researchers meet informally to discuss, argue, and brainstorm, thus providing a stimulus for "out of the box" ideas in settings conducive to "risky" thoughts. At workshops and summer schools, perhaps focused on a single challenge area, participants can explore new ideas without the pressure of producing immediate results. Such events often serve as the catalyst for new ideas and collaborations.

\subsection{Building effective connections with science and engineering.}

A key metric for success of the Applied Mathematics program is contributing mathematical analyses and algorithms that advance DOE's mission. Progress in this direction is accelerated when mathematicians work closely with application scientists and engineers who are connected to mission needs, since new insights frequently arise from a fortuitous combination of multiple perspectives. The strategy of forming and funding teams of collaborators from different disciplines has been highly successful in addressing several complex DOE problems. However, depending on context, different organizational modes are needed.

In some cases, the scientific issues are sufficiently well understood so that the mathematical research is limited to development of models that are both physically accurate and computationally tractable. The most appropriate operational strategy is then likely to be the formation of partnerships between mathematicians and scientists, jointly funded by their respective sponsors, to produce a mathematical model tailored to that application area. 


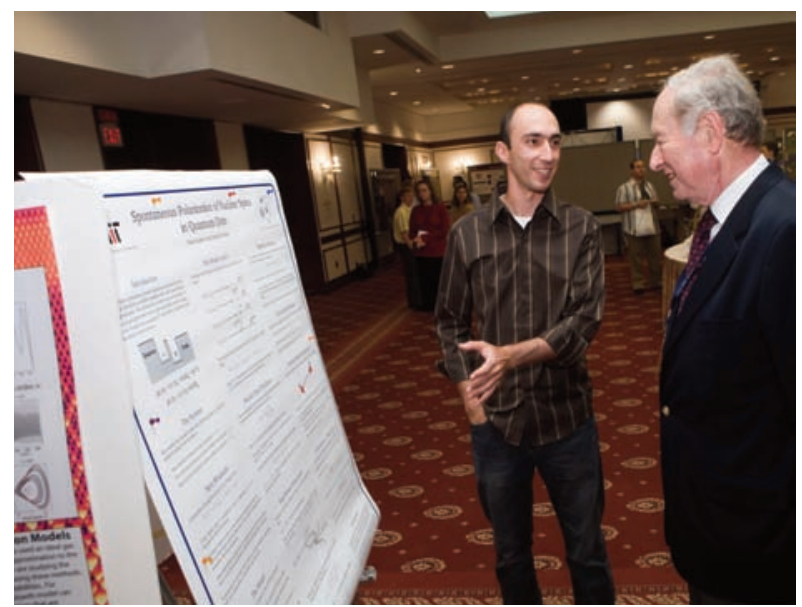

FIGURE 12. DOE's Computational Science Graduate Fellowship Program provides support to help train next generation of computational scientists

In other situations, however, the research issues are primarily mathematical, such as when the science is less well-developed or the mathematical approaches are likely to be relevant to a broad set of application areas. For such problems, mathematicians should ideally participate in every step of the problem solution process, including the initial formulation of a model as well as design of algorithms and software. When the creation of new mathematics is the most essential element in research involving mathematicians and application scientists, it may be most effective for the mathematics sponsor (in this case, the Office of Advanced Scientific Computing Research) to provide all of the research support.

\subsection{Leveraging the expertise in National Laboratories and universities}

The Applied Mathematics program should take maximum advantage of mathematics research talent, keeping in mind the differences between the National Laboratories and academia. With full-time professional research staff who often spend significant fractions of their career at a single institution, Laboratories are well positioned to pursue largescale research efforts, especially those involving collaboration among a group of researchers over a long period of time. The Laboratories are also ideal environments to develop and maintain high-quality mathematical software libraries, which require consistent long-term attention.

The institutional mobility of researchers in academia, including PhD students and postdoctoral researchers, tends to be much larger. While a faculty research advisor can focus on a single research area for many years, much of the research "product" in universities consists of self-contained efforts completed by a single student or postdoctoral researcher. The research produced by these latter junior researchers often includes significant new ideas.

Because an increase in multidisciplinary research will be essential for driving future advancements in applied mathematics for the DOE mechanisms that encourage multidisciplinary activities, both at Laboratories and at universities, will be important. To enhance the ties between Laboratories and academia, mechanisms could be devised to provide incentives for academic investigators to join multidisciplinary collaborative teams and Laboratory partnerships. Since the National Laboratories are responsible for executing much of DOE's "big science", the Laboratories could organize multi-institutional activities that bring mathematicians together with scientists and engineers to work together on scientific areas of particular importance to DOE.

\subsection{Connecting applied mathematics and advanced computing.}

Although the primary focus of the Applied Mathematics program is foundational mathematics, an element differentiating DOE's program from others is its longstanding commitment to the mathematical and computational underpinnings of largescale scientific discovery through high-performance computing. A crucial link between mathematics and applications is high-quality mathematical software that embodies the latest models and algorithms and is also designed to run efficiently on leading-edge computing platforms. Effective mechanisms for producing, maintaining and enhancing this software are an essential part of applied mathematics within DOE. 


\section{Epilogue}

This report has provided an analysis of the DOE's needs for new applied, computational and statistical mathematical developments in order to support the science and engineering-based solutions to the problems of critical national importance that DOE must address in the future. Through effective management and thoughtful direction, the DOE Applied Mathematics program can continue its half-century legacy in making significant, enduring advances in applied mathematics that will enable future scientific discovery through computational science. This program will make significant advances in the predictive modeling, simulation and analysis of complex natural and engineered systems in support of the DOE's energy, environmental and national security missions.

Effective policy-making on issues of critical national importance will be supported by innovative, often transformational mathematical advances produced by this program. 


\section{References}

1. U. S. Department of Energy Strategic Plan, 2006. http://www.energy.gov/about/strategicplan.htm

2. Keyes, D., Colella, P., Dunning Jr., T., and Gropp, W., (Eds.): A Science-Based Case for Large-Scale Simulation - Volume 1: Department of Energy - Office of Science Workshop Report, July 2003. http://www.pnl.gov/scales/

3. Keyes, D., Colella, P., Dunning, Jr., T., and Gropp, W. (Eds.); A Science-Based Case for LargeScale Simulation - Volume 2: Department of Energy - Office of Science Workshop Report, September 2004. http://www.pnl.gov/scales/

4. Oden, J. T. (Ed.); Simulation-Based Engineering Science: Revolutionizing Engineering Science through Simulation - Report of the National Science Foundation Blue Ribbon Panel on Simulation-Based Engineering Science, February 2006. http://www.nsf.gov/pubs/reports/sbes_ final_report.pdf

5. Bashor, J. and Hules, J. (Eds.); Scientific Discovery: A progress report on the U.S. Department of Energy's Scientific Discovery through Advanced Computing (SciDAC) Program, Office of Science ,Office of Advanced Scientific Computing Research of the U.S. Department of Energy, 2006. http://www.scidac.gov/img_ results/FinalSciDACreport.pdf

6. Finck, P., Keyes, D., and Stevens, R. (Eds.); Workshop on Simulation and Modeling for Advanced Nuclear Energy Systems, Department of Energy - Office of Science Workshop Report, August 2006. www.er.doe.gov/ascr/Misc/gnep06-final.pdf

7. Dalhburg, J. (Ed.); Fusion Simulation Project: Integrated Simulation \& Optimization of Fusion Systems, U.S. Department of Energy - Office of Science, Final report of the Fusion Energy Science Advisory Committee, Integrated Simulation and Optimization of Magnetic Fusion Systems Subcommittee, DOE/SC-0073, November 2002. www.ofes.fusion.doe.gov/ More_HTML/ FESAC/FESAC1102/Dahlburg.pdf
8. Kritz, A., and Keyes, D. (Eds.); Fusion Simulation Project Workshop Report: U. S. Department of Energy - Office of Science Workshop Report, July 2007, http://www.sc.doe.gov/ofes/More_ HTML/FESAC/FESAC07/FSP_report_070702d.pdf

9. Office of Clean Coal Strategic Plan, Office of the Assistant Secretary for Fossil Energy - U.S. Department of Energy, September 2006

10. Report of the Computational Subsurface Sciences Workshop, U.S. Department of Energy - Office of Science Workshop report, January 2007, http://subsurface2007.labworks.org/report/

11. Breaking the Biological Barriers to Cellulosic Ethanol: A Joint Research Agenda, U.S. Department of Energy - Office of Science Workshop report, DOE/SC-0095, June 2006, http://genomicsgtl.energy.gov/biofuels/b2bworkshop.shtml

12. McCurdy, C. W., Stechel, E., Cummings, P., Hendrickson, B., and Keyes, D. (Eds.); Theory and Modeling in Nanoscience, U.S. Department of Energy - Office of Science Workshop Report, 2002, http://repositories. cdlib.org/lbnl/LBNL-50954/

13. Hendrickson, B. A., and Wright, M. H., (Eds.); Mathematical Research Challenges in Optimization of Complex Systems, U. S. Department of Energy - Office of Science Workshop Report, December 2006, http://www.sc.doe.gov/ascr/Research/AM/ ComplexSystemsWorkshopReport.pdf

14. Mount, R. P. (Ed.); The Office of Science DataManagement Challenge, U.S. Department of Energy - Office of Science Workshop Report, November 2004, http://www.science.doe.gov/ ascr/ProgramDocuments/Final-report-v26.pdf

15. Lax, P. D., Hyperbolic Systems of Conservation Laws and the Mathematical Theory of Shock Waves, Society for Industrial and Applied Mathematics, 1973.

16. Colella, P., Hou, T. and Petzold, L. (Eds.); Report of the First Multiscale Mathematics Workshop: First Steps toward a Roadmap, U. S. Department 
of Energy - Office of Science Workshop Report, August 2004, http://www.sc.doe.gov/ascr/ Research/AM/MultiscaleMathWorkshop1.pdf

17. Estep, D., Shadid, J. N., and Tavener, S. J., (Eds.); Final Report: Second DOE Workshop on Multiscale Problems, Broomsfield, Colorado, U. S. Department of Energy - Office of Science Workshop Report, October 2004, http://www. sc.doe.gov/ascr/Research/AM/MultiscaleMathW orkshop2.pdf
18. Dolbow, J., Khaleel, M. A., Mitchell, J., (Eds.); Multiscale Mathematics Initiative: A Roadmap, U.S. Department of Energy - Office of Science Workshop Report, December 2004. http://www. sc.doe.gov/ascr/Research/AM/MultiscaleMathWo rkshop3.pdf

19. Experiments in International Benchmarking of U.S. Research Fields, the National Academies Press, 2000, page 54. 